OPEN ACCESS

Edited by:

Sabine Elowe

Laval University, Canada

Reviewed by:

Mónica Pradillo,

Complutense University of Madrid,

Spain

Daniel Leslie Fisher,

Centre National de la Recherche

Scientifique (CNRS), France

*Correspondence:

Adele L. Marston adele.marston@ed.ac.uk Katja Wassmann

katja.wassmann@upmc.fr

Specialty section

This article was submitted to

Cell Growth and Division,

a section of the journal

Frontiers in Cell and Developmental

Biology

Received: 02 October 2017 Accepted: 28 November 2017 Published: 13 December 2017

Citation:

Marston AL and Wassmann K (2017) Multiple Duties for Spindle Assembly

Checkpoint Kinases in Meiosis.

Front. Cell Dev. Biol. 5:109.

doi: 10.3389/fcell.2017.00109

\section{Multiple Duties for Spindle Assembly Checkpoint Kinases in Meiosis}

\author{
Adele L. Marston ${ }^{1 *}$ and Katja Wassmann ${ }^{2,3 *}$ \\ ${ }^{1}$ Wellcome Centre for Cell Biology, Institute for Cell Biology, University of Edinburgh, Edinburgh, United Kingdom, ${ }^{2}$ Sorbonne \\ Universités, UPMC Univ Paris 06, Institut de Biologie Paris Seine, UMR7622, Paris, France, ${ }^{3}$ Centre National de la \\ Recherche Scientifique, Institut de Biologie Paris Seine, UMR7622 Developmental Biology Lab, Paris, France
}

Cell division in mitosis and meiosis is governed by evolutionary highly conserved protein kinases and phosphatases, controlling the timely execution of key events such as nuclear envelope breakdown, spindle assembly, chromosome attachment to the spindle and chromosome segregation, and cell cycle exit. In mitosis, the spindle assembly checkpoint (SAC) controls the proper attachment to and alignment of chromosomes on the spindle. The SAC detects errors and induces a cell cycle arrest in metaphase, preventing chromatid separation. Once all chromosomes are properly attached, the SAC-dependent arrest is relieved and chromatids separate evenly into daughter cells. The signaling cascade leading to checkpoint arrest depends on several protein kinases that are conserved from yeast to man. In meiosis, haploid cells containing new genetic combinations are generated from a diploid cell through two specialized cell divisions. Though apparently less robust, SAC control also exists in meiosis. Recently, it has emerged that SAC kinases have additional roles in executing accurate chromosome segregation during the meiotic divisions. Here, we summarize the main differences between mitotic and meiotic cell divisions, and explain why meiotic divisions pose special challenges for correct chromosome segregation. The less-known meiotic roles of the SAC kinases are described, with a focus on two model systems: yeast and mouse oocytes. The meiotic roles of the canonical checkpoint kinases Bub1, Mps1, the pseudokinase BubR1 (Mad3), and Aurora B and C (Ipl1) will be discussed. Insights into the molecular signaling pathways that bring about the special chromosome segregation pattern during meiosis will help us understand why human oocytes are so frequently aneuploid.

Keywords: meiosis, spindle assembly checkpoint, Mps1, Bub1, BubR1/Mad3, Aurora B-like kinases, cohesin protection, chromosome congression

\section{GENERAL INTRODUCTION INTO MEIOSIS}

All sexually reproducing organisms rely on a specialized form of cell division known as meiosis, the defining feature of which is the generation of gametes with half the number of chromosomes of the parental cell. In contrast to the mitotic divisions, where ploidy is maintained by alternate rounds of DNA replication and chromosome segregation; during meiosis, replicated chromosomes are segregated twice successively, reducing ploidy by half. In most organisms, including humans and the model organisms discussed in this review, maternal and paternal chromosomes (homologs) are segregated during meiosis I, while the copies generated during DNA replication (sister chromatids) 
are segregated only during meiosis II, reminiscent of mitosis (reviewed in Marston and Amon, 2004). Perhaps as a result of the added complexity of successively segregating chromosomes, meiosis is highly error-prone. In human females, up to $\sim 30 \%$ of all female gametes (oocytes/eggs) carry the incorrect number of chromosomes (Hassold and Hunt, 2001). Although such aneuploidy is a major cause of miscarriages, infertility and birth defects, the underlying molecular lesions are not wellunderstood. In part, this is due to a lack of knowledge about the pathways that orchestrate the meiotic divisions. Indeed, compared to mitosis, where intensive research over many years has shaped our understanding, our mechanistic knowledge of meiosis is less developed. In recent years, however, it has become apparent that many regulators of the mitotic cell cycle take on increased significance during meiosis. This is to some extent because meiosis is especially reliant on the canonical processes they control, but also because mitotic regulators gain novel functions during meiosis, in part through establishment of distinct interactions. This is particularly true for cell cyclerelevant kinases, which adopt a wide range of novel and canonical functions to control meiosis. Here, we focus on kinases of the central surveillance pathway in mitotic cells: the spindle assembly checkpoint (SAC) and review their roles in meiosis.

\section{Model Systems to Study Meiotic Divisions}

Our knowledge of meiosis is derived from a rich array of model organisms. Pathways of homolog recognition and meiotic recombination have particularly benefited from a long history of complementary studies in diverse organisms. We focus here on studies of the SAC kinases in the yeasts Saccharomyces cerevisiae and Schizosaccharomyces pombe, and mouse oocytes. For clarity, gene names relating to different organisms are given in the order: S. cerevisiae/S. pombe/mouse.

\section{Budding Yeast and Fission Yeast}

Unicellular fungi, in particular the budding yeast, Saccharomyces cerevisiae, and the fission yeast, Schizosaccharomyces pombe, have provided a wealth of basic information relating to chromosome segregation during meiosis. S. cerevisiae undergoes a vegetative (mitotic) cycle as a haploid, existing in two mating types (sexes), known as a and alpha, which can mate to form an a/alpha diploid, which can also undergo a vegetative cycle (Figure 1). Upon starvation, diploids will undergo meiosis to generate four haploid spores. S. pombe similarly exists in two haploid sexes $(\mathrm{h}+$ and $\mathrm{h}-$ ) that undergo conjugation and meiosis to generate four haploid spores. The ability to isolate both haploids and diploids of yeasts enables ease of genetic manipulation. One advantage of both yeasts for studying meiosis is that all four products of

Abbreviations: SAC, Spindle Assembly Checkpoint; Bub1, 3, Budding uninhibited by Benomyl 1, 3; BubR1, Bub1 related; Mad1-3, Mitotic arrest deficient 1-3; Mps1, Monopolar Spindles 1; Ipl1, Increase-in-ploidy 1; Ark1, Aurora-related kinase 1; SPB, Spindle Pole Body; APC/C, Anaphase Promoting Complex/ Cyclosome; MPF, M-phase Promoting Factor; CSF, Cytostatic Factor; GVBD, Germinal Vesicle Breakdown; PKA, Protein Kinase A; Cdk1, 2, Cell division kinase 1, 2; Cdc20, Cell division cycle 20; Zp3, Zona pellucida 3; Knl1, Kinetochore null 1; MCC, Mitotic Checkpoint Complex; Sgo1, 2, Shugoshin 1,2; PP2A, Protein phosphatase 2A; H2A, Histone H2A; Mph1, Mps1p-like pombe homolog; Wapl, Wings apart-like protein homolog. meiosis can be isolated, so that chromosome segregation can be recapitulated. Furthermore, a wide range of genetic tools exist. This includes the ability to label individual chromosomes with fluorescent markers (Straight et al., 1996; Michaelis et al., 1997) and methods to highly synchronize cell populations (Bähler et al., 1991; Carlile and Amon, 2008; Kakui et al., 2011; Cipak et al., 2012; Chia and van Werven, 2016). Yeast also have the advantage that large quantities can easily be grown for biochemical analyses, yet they are equally amenable to single cell live cell imaging.

\section{Mouse Oocytes}

Most of our knowledge on the regulation of meiotic divisions in mammals has been obtained from mouse oocytes. Male division in meiosis cannot currently be dynamically followed in the same manner as female meiosis, therefore we focus here on female meiosis. Mammalian oocytes are generated in the female embryo from germinal cells that form the primordial follicle. Until sexual maturity, oocytes are maintained arrested in prophase I untildepending on the species-a variable number are hormonally stimulated to grow and enter the first meiotic division (El Yakoubi and Wassmann, 2017). To study murine oocyte cell division in meiosis I and II, mature prophase I arrested oocytes that have finished their growth phase can be obtained from adult, female mice. Oocytes are usually harvested in medium that maintains the prophase arrest by inhibiting the signaling pathway inducing a rise in Cdk1 associated kinase activity, such as for example medium containing dbcAMP, which maintains the kinase PKA active, preventing Cdk1 activation. This is necessary, because mammalian oocytes (unlike Xenopus laevis oocytes for example) spontaneously enter the first meiotic division upon removal of the follicle cells surrounding them. Mouse oocytes that have been cleaned from follicle cells are then released into medium allowing maturation. Under normal culture conditions, oocytes undergo Germinal Vesicle breakdown (GVBD), which is visible under the microscope, in a very synchronized manner, 45-90 min after release. The metaphase-to-anaphase transition of the first meiotic division takes place around $7-10 \mathrm{~h}$ after GVBD, depending on the mouse strain that is being used. Exit from meiosis I is visible due to the extrusion of a small Polar Body (PB). Oocytes then progress into meiosis II, where they remain arrested in metaphase II to await fertilization. This arrest point is called CSF, or Cytostatic Factor arrest (Perry and Verlhac, 2008). It is only upon fertilization that oocytes finalize the second meiotic division in vivo, but in vitro cultured oocytes in metaphase II can be artificially "activated" to undergo the metaphase II-to-anaphase II transition. The high synchrony and temporal resolution of the meiotic divisions permits harvesting pools of oocytes at all meiotic cell division stages for fixation or preparation of cell extracts, or their observation by live imaging.

Mouse oocytes are transparent and can be injected with fluorescent proteins to follow, for example, chromosome movements or the localization of kinetochore proteins. Conditional knock-out approaches using oocyte-specific promoters such as Zona pellucida 3 (Zp3) to express Cre recombinase, are successful to knock-out genes during oocyte growth before entry into meiosis I (Lewandoski et al., 1997; Lan et al., 2004). This approach can be used for gene products that 

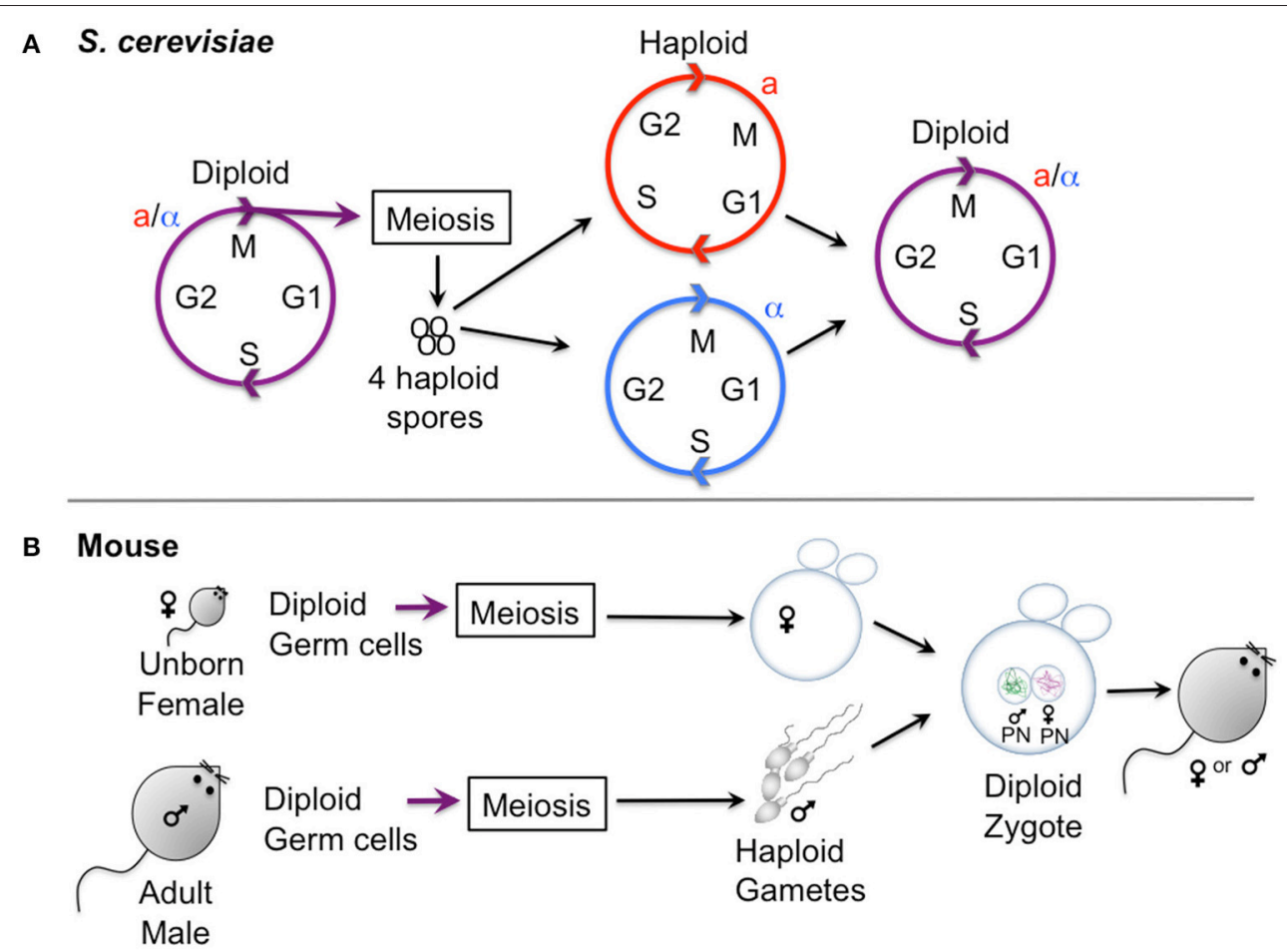

FIGURE 1 | Gametogenesis in yeast and female mice. (A) Outline of the lifecycle of the budding yeast S. cerevisiae, which can propagate vegetatively through mitotic division in both its haploid and diploid form. Haploid yeast of opposite mating types (a and alpha) mate and undergo conjugation to generate a zygote, upon which nuclei fuse (karyogamy). Starvation of diploid cells triggers meiosis, culminating in the production of four haploid spores. Note that the lifestyle of $S$. pombe is similar, except that meiosis and sporulation occur directly after karyogamy so that diploid cells are short-lived. (B) Oocyte differentiation in female mice. Gametes are generated from diploid germ cells, in the female embryo before birth for oocytes, and in the adult male for spermatocytes. After hormonal stimulation in adult females, some oocytes that are arrested in prophase I undergo a growth phase. During the menstrual cycle usually one (humans) or several (mouse) oocytes are induced to undergo meiosis I and enter meiosis II, where they will remain arrested to await fertilization with a male gamete that has already finished meiosis I and II and has progressed into G1 phase. Oocytes exit meiosis II after fertilization, and the male and female pronucleus fuse to form the diploid zygote, the first cell of the embryo.

are otherwise essential for cell division, and that are not stable throughout the different meiotic stages before entry into meiosis I. Oocytes can be fixed for immunostaining or chromosome spreads, but they are not suitable for more sophisticated biochemical assays, due to the small oocyte count per mouse and the low amount of protein per oocyte. Alternative model systems have to be used for purification of proteins or activity assays.

\section{Mitotic and Meiotic Divisions: Specialization of the Chromosome Segregation Machinery during Meiosis}

The generation of gametes with half the chromosome content to somatic cells requires the remodeling of the chromosome segregation machinery. It is useful to consider mitotic chromosome segregation before discussing the modifications that are superimposed on the canonical machinery for execution of the meiotic divisions.

\section{Mitosis}

Following DNA replication during mitosis, the two newly duplicated sister chromatids are held tightly together by a ringshaped protein complex, called cohesin, which is postulated to provide this cohesion by entrapping the sister chromatids topologically within (reviewed in Nasmyth, 2011). In mammals, but not yeast, the bulk of cohesin is removed from chromosome arms due to the action of Wapl, acting in the so-called prophase pathway. However, a fraction of cohesin is retained, primarily at the centromeres to ensure that sister chromatids remain associated. Pericentromeric shugoshin (Sgo1) protein, counteracts Wapl1 activity to ensure retention of cohesin in this region. At metaphase, the sister kinetochores assembled on the centromeres attach to spindle microtubules that emanate from opposite poles of the cell and cohesin counteracts the resultant pulling forces. The SAC and error correction machinery (see below) together monitor this attachment, delaying anaphase onset until all chromosomes have achieved the stable biorientation of sister chromatids. Once this has occurred, the SAC is satisfied and the enzyme, separase, becomes active and cleaves all remaining cohesin along the entire length of chromosomes, destroying the links between sister chromatids and initiating their poleward movement.

\section{Meiosis}

The segregation of homologs during meiosis I, followed by sister chromatids during meiosis II requires that the canonical chromosome segregation machinery is adapted. As in mitosis, 
chromosomes are replicated and cohesin is established between them. Subsequently, two consecutive chromosome segregation events occur. In addition to poorly understood changes to cell cycle controls, which not only ensure that meiosis I is followed not by interphase, but by meiosis II, the configuration of chromosomes is different in 3 major ways (Figure 2) (Marston and Amon, 2004). First, homologous chromosomes must be linked together to enable them to attach to microtubules in a tension-generating configuration. This requires homologs to pair with their partner and, in most organisms, they will undergo crossover recombination to produce chiasmata and generate a bivalent (consisting of 4 chromatids, Figure 2) (reviewed in Hunter, 2015). Chiasmata, together with cohesion between sister chromatids on chromosome arms distal to the chiasmata, hold the homologs together in this bivalent. This is because, once DNA repair is complete, a chiasma constitutes two DNA strands, which cross, but are not physically associated themselves. Rather, the recombinant sister chromatid (blue and pink in Figure 2) makes cohesive contacts not only with its identical sister chromatid but, also distal to the chiasmata, with its homologous chromatid, thereby linking the homologs and stabilizing the bivalent. Second, in contrast to mitosis, or meiosis II, where sister kinetochores attach to microtubules from opposite poles (sister kinetochore bi-orientation); during meiosis II, sister kinetochores attach to microtubules from the same pole (co-orientation, also called mono-orientation). In budding yeast, maize and flies, fusion of sister kinetochores appears to underlie their co-orientation (Goldstein, 1981; Li and Dawe, 2009; Sarangapani et al., 2014), however recent data suggest that this may not be the case in human oocytes (Patel et al., 2015; Zielinska et al., 2015). Third, cohesin, the ring shaped complex which is established during DNA replication to hold sister chromatids together, is lost in two steps during meiosis. During meiosis I, cohesin is proteolytically cleaved along chromosome arms by the enzyme separase. Because sister chromatid cohesion on chromosome arms distal to chiasmata is the only thing holding the homologs together, this triggers their segregation to opposite poles. However, cohesin is maintained in the region surrounding the centromeres, which ensures that sister chromatids remain linked until meiosis II. Protection of pericentromeric cohesin from separase activity requires a complex of shugoshin and protein phosphatase $2 \mathrm{~A}$, which are localized in the pericentromere and here act to prevent the proteolytic loss of cohesin, rather than Wapl-dependent removal as in mammalian mitosis (reviewed in Marston, 2015; specific aspects discussed below).

It is also important to note here that mammalian oocytes, and those of other animal species such as Drosophila, also encounter

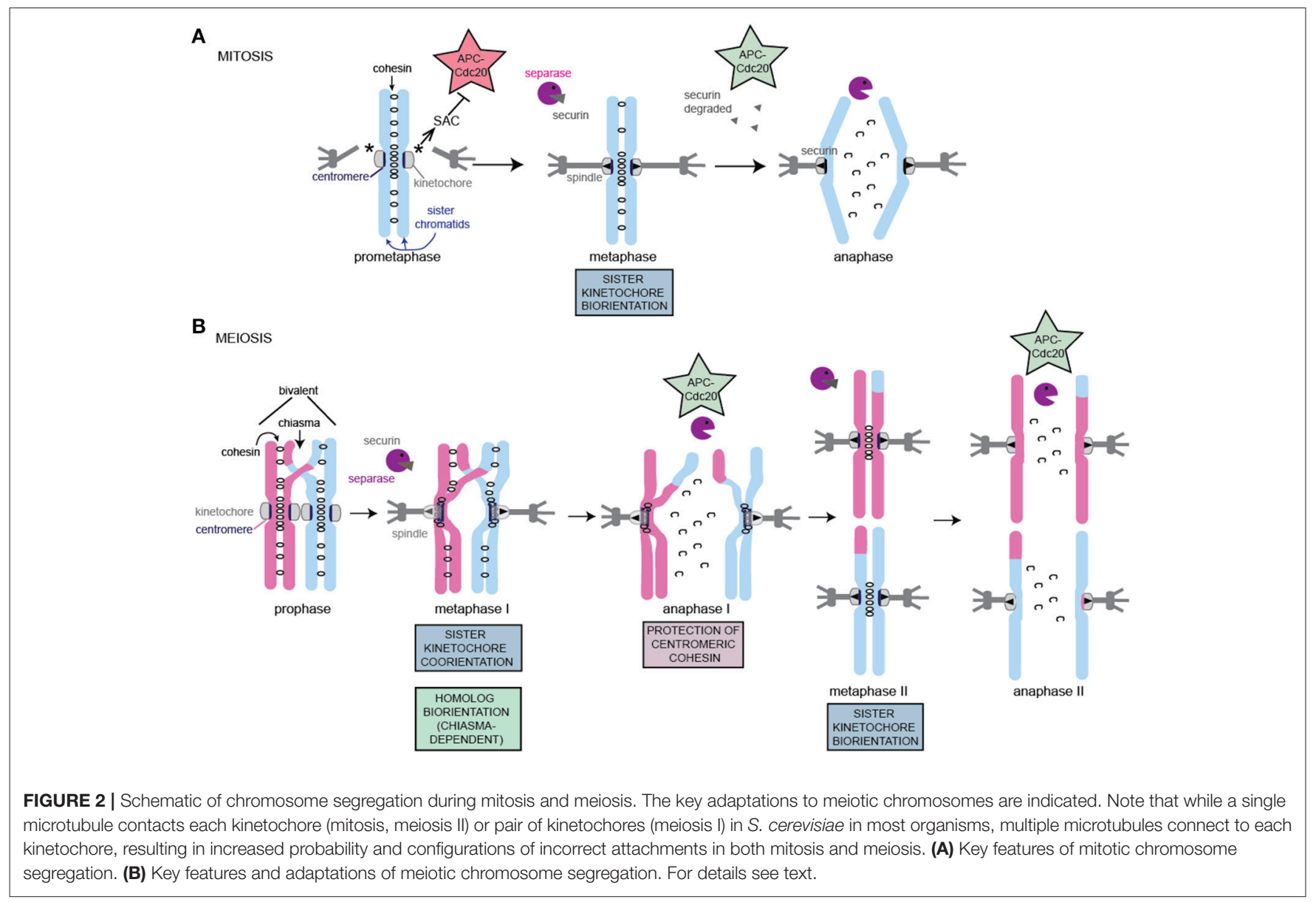


additional challenges to segregate chromosomes compared to yeasts in building a bipolar spindle and attaching kinetochores to microtubules. This is because oocytes are acentrosomal, and instead the chromosomes themselves organize the establishment of a bipolar spindle (Ohkura, 2015). Furthermore, the number of microtubule binding sites on each kinetochore is greater in mammalian oocytes, increasing the complexity of kinetochoremicrotubule attachments (S. cerevisiae, in contrast, have only a single microtubule-binding site per kinetochore; Winey et al., 1995).

\section{Consequences of Mis-Segregation in Meiosis}

Two haploid gametes fuse to give rise to a diploid cell -the zygote- which is the first cell of the future embryo. If one of the gametes does not harbor the correct chromosome count, the resulting embryo will be aneuploid. Most aneuploidies are not viable, and in humans only certain trisomies, or monosomies of the sex chromosomes, are viable. Therefore, human aneuploid embryos are spontanously aborted in the first trimester and increased aneuploidy rates in meiosis affect pregnancy outcome. In humans, the high aneuploidy rate in female meiosis has important consequences for achieving a successful pregnancy. Mis-segregation rates in meiosis increase sharply with maternal age, with women closer to menopause having a risk of more than $30 \%$ of being pregnant with a trisomic baby (Hassold and Hunt, 2001; El Yakoubi and Wassmann, 2017).

The reasons for the high error rate in meiotic chromosome segregation are multiple: Oocytes are not replenished with age, and before entry into the first meiotic division they may have been arrested in prophase for several decades. It has been shown in mice that there is no turn-over of cohesins during the extended prophase I arrest where oocytes await hormonal stimulation to grow and divide (Revenkova et al., 2010; Tachibana-Konwalski et al., 2010). Cohesins deteriorate with age and this leads to less solid connections between sister chromatids and as a consequence, weakening and even loss of chiasmata (Chiang et al., 2010; Lister et al., 2010). Once oocytes enter meiosis I, sister chromatids which are not held together by chiasmata cannot attach correctly and will biorient (Jessberger, 2012). The SAC may not be able to distinguish monopolar attachments of sister kinetochores of a bivalent from the bipolar attachment of a single chromosome, as both generate tension that will stabilize end-on attachments. Additionally, it was shown in human oocytes that recruitment of the SAC factors Bub1 and BubR1 to kinetochores decrease with age indicating that the SAC is less able to recognize missing attachments and to induce a cell cycle arrest (Lagirand-Cantaloube et al., 2017). This decrease in addition to the inherent leakiness of the meiotic checkpoint, which requires several unattached/tensionless kinetochores to mount a robust SAC dependent delay, could additionally lead to the observed increased error rate in oocytes of aged mothers.

Loss of cohesin with age not only leads to loss of chiasmata resulting in biorientation of unpaired chromosomes in meiosis I, but can also allow the precocious separation of sister chromatids. In mice it was shown that recruitment of the centromeric cohesin protector, Sgo2, to the centromere region also diminishes with age, and this has been correlated with premature loss of cohesin and therefore separation of some sister chromatids in meiosis I instead of in meiosis II (Lister et al., 2010). Such uncohered sister chromatids cannot be separated correctly in meiosis II, however, a rescue mechanism, whereby sister chromatids that have undergone recombination are preferentially segregated into the oocytes, rather than the polar body, has recently been discovered in humans (Ottolini et al., 2015). This could represent a form of "meiotic drive," a phenomenon whereby certain traits are preferentially transmitted to the offspring.

\section{Spindle Assembly Checkpoint Control}

The specialised chromosome segregation pattern in meiosis also requires the adaptation of control mechanisms that regulate cell divisions in somatic cells. One control mechanism of significant importance during meiotic cell division in mammalian oocytes is the spindle assembly checkpoint or SAC, which ensures that chromosome segregation takes place only after correct end-on kinetochore microtubule attachments to both poles have been achieved (Wassmann et al., 2003; Homer et al., 2005; Niault et al., 2007; McGuinness et al., 2009; Hached et al., 2011). Molecular details on checkpoint response have been mainly discovered in mitotic cells of different model organisms, thus mitotic SAC control will be shortly introduced below before discussing the specificities of this checkpoint in meiosis.

In mitosis, paired sister chromatids have to be faithfully segregated into two daughter cells. Failures in the correct partitioning of the genetic material will lead to the generation of aneuploid daughter cells which harbor an incorrect number of chromosomes. Therefore it is pivotal that separase is not activated to remove cohesin before all sister chromatids are correctly attached with their kinetochores to the bipolar spindle. It is the job of the SAC to ensure that anaphase onset takes place only when stable end-on attachments that are under tension are present at each kinetochore. Tension applied by the opposite poles of the spindle further stabilizes attachments. In short, the SAC recognizes the presence of kinetochores that harbour unoccupied microtubule binding sites, and if this is the case, delays the metaphase-to-anaphase transition by inhibiting the anaphase promoting complex/cyclosome (APC/C) coupled to its activator, $\mathrm{Cdc} 20 . \mathrm{APC} / \mathrm{C}^{\mathrm{Cdc} 20}$ activation will result in the degradation of the separase inhibitor, securin, resulting in cohesin cleavage and is essential for chromatid segregation in mitosis, and chromosome segregation in meiosis I in S. cerevisiae, Xenopus and mouse oocytes (Herbert et al., 2003; Terret et al., 2003; Lee et al., 2004; Kudo et al., 2006; Zhang et al., 2008; Note that this was initially controversial in Xenopus, see discussion in Zhang et al., 2008). APC/C $\mathrm{C}^{\mathrm{Cdc} 20}$ inhibition by the SAC depends on the phosphorylation of the SAC components Bub1, Mad1, the kinetochore protein Knl1 by the kinase Mps1, and binding of Mad2 to attachment sites on the kinetochore that are devoid of stable microtubule binding. Mps1 localizes on and off the microtubule binding sites on the unattached kinetochores as long as there are no stably bound microtubules, thereby maintaining SAC activity. Continuous cycling of cytosolic Mad2 between active and inactive conformations by binding 
to Mad1/Mad2 complexes that are more stably associated with unattached kinetochores leads to the generation of the diffusible MCC (mitotic checkpoint complexes), which binds to $\mathrm{APC} / \mathrm{C}^{\mathrm{Cdc} 20}$ to induce metaphase arrest (for more details see excellent reviews (Khodjakov and Pines, 2010; Musacchio, 2015; Sacristan and Kops, 2015). In S. cerevisiae and Drosophila, SAC control is essential only upon conditions when proper microtubule-kinetochore attachments are perturbed, for example after treatment with microtubule depolymerizing drugs or the introduction of mutants affecting establishment of a proper spindle (Hoyt et al., 1991; Li and Murray, 1991; Buffin et al., 2007). In mammals, the checkpoint is active during each cell cycle as cells enter mitosis and progress through prometaphase, and prevents accelerated progression through early steps of mitosis (Taylor and McKeon, 1997; Gorbsky et al., 1998; Dobles et al., 2000; Kalitsis et al., 2000). SAC control is therefore essential to generate euploid daughter cells under unchallenged conditions. The SAC is gradually shut off as cells progress into metaphase and end-on attachments are established. In the absence of a functional $\mathrm{SAC}$, progression through mitosis is accelerated (Meraldi et al., 2004; Tighe et al., 2008; Sliedrecht et al., 2010). As long as anaphase onset did not occur, the checkpoint can be re-activated, and cells re-establish a metaphase arrest (Collin et al., 2013; Kamenz and Hauf, 2014; Vázquez-Novelle et al., 2014).

Once separase becomes active and removes cohesin at anaphase onset, attachments are no longer under tension. SAC activity depends on elevated Cyclin B-Cdk1 levels, therefore once anaphase onset occurs and Cyclin B1 becomes degraded, the SAC can no longer be re-activated. Hence, the metaphase-to-anaphase transition is regulated by a bistable switch through two feedback loops, ensuring the irreversibility of anaphase onset ( $\mathrm{He}$ et al., 2011).

On a molecular level it is known that the cell cycle arrest in metaphase upon SAC activation is brought about by the generation of the MCC, which consists of the core SAC components Mad2, BubR1 and Bub3, and the APC/C activator Cdc20 (Fraschini et al., 2001; Sudakin et al., 2001; Tang et al., 2001). The MCC prevents ubiquitination and hence degradation of two key APC/C substrates at the metaphase-to-anaphase transition, namely Cyclin B1 and securin. In vertebrates, both proteins keep separase inactive, and their stabilization upon checkpoint activation thus prevents cohesin removal in metaphase, and anaphase onset. Several kinases and one pseudokinase are involved in SAC control (Sacristan and Kops, 2015), with the dual specificity kinase Mps1 playing a pivotal role, which will be outlined in detail below. The kinase Bub1 is essential for SAC functioning, but independently of its kinase activity. Mammalian BubR1 is a pseudo-kinase, homologous to yeast Mad3 and an essential component of the MCC.

Working in conjunction with the SAC is the "error correction" system, which detects tension-less kinetochoremicrotubule attachments and severs them, thereby providing a further opportunity for correct, tension-generating attachments to be established. Aurora B kinase activity is central to this process, working by directly phosphorylating components at the interface of the microtubule-kinetochore interaction to abolish it (see Krenn and Musacchio, 2015 for a review on the various kinetochore substrates). This in turn, creates unattached kinetochores, which activate the SAC. Whether Aurora B is also part of the SAC signaling cascade per se is still controversial (Santaguida et al., 2011; Gurden et al., 2016). Because Aurora B participates in proper Mps1 localization, it is at least indirectly required for SAC signaling (Maldonado and Kapoor, 2011; Saurin et al., 2011).

\section{SAC Control in Meiosis}

The meiotic divisions in oocytes are highly error prone and have led to speculations on the inefficiency of SAC control in meiosis. In S. cerevisiae, loss of Mad2 leads to an acceleration of meiosis I with premature $\mathrm{APC} / \mathrm{C}$ activation, and the generation of aneuploid spores, due in part to the mis-segregation of homologs during meiosis I (Shonn et al., 2000, 2003; Cheslock et al., 2005; Lacefield and Murray, 2007; Tsuchiya et al., 2011). Given that MAD2 is not essential for viability in S. cerevisiae, this indicates a much greater requirement for the SAC in the accurate segregation of homologs during meiosis I than in mitosis. In contrast, Xenopus laevis oocytes have been reported to divide without being able to mount a detectable checkpoint delay, and despite this the error rate of chromosome segregation is not worse than in mammalian oocytes, which can induce a SAC delay (Shao et al., 2013; Liu et al., 2014). In Drosophila oocytes, the SAC components Mps1 and BubR1 are required for correct chromosome segregation in meiosis I (Gilliland et al., 2005; Malmanche et al., 2007), but according to one study, without detectable influence on APC/C activity (Batiha and Swan, 2012), and therefore potentially independently of the SAC. However, SAC proteins are present in both Drosophila and X. laevis oocytes so it remains possible that they provide some function during meiosis in these organisms, too.

On the contrary, it is well established that mammalian oocytes possess a functional checkpoint leading to a detectable delay in anaphase I onset, when activated, even though the meiotic cell divisions are highly error prone (Wassmann et al., 2003; Homer et al., 2005; Niault et al., 2007; Hassold and Hunt, 2009; McGuinness et al., 2009; Hached et al., 2011). Mice harboring a conditional knock-out of any of the essential SAC components are sterile, because meiosis I is significantly accelerated and chromosome missegregations occur at elevated rates. The checkpoint kinases Mps1, Bub1, and the pseudokinase BubR1 are, as in mitosis, essential for SAC functioning (McGuinness et al., 2009; Hached et al., 2011; Touati et al., 2015). The meiotic checkpoint in oocytes is rather leaky (Gui and Homer, 2012; Lane et al., 2012; Sebestova et al., 2012), which may be related to the huge size of the oocyte on the one hand, and the specificities of the meiotic divisions as far as kinetochore orientation is concerned, on the other hand. The molecular details of SAC control in meiosis compared to mitosis are still not entirely clear, as well as the question of what kind of errors are detected in meiosis compared to mitosis. Because we want to focus here on the less-known roles of checkpoint kinases in meiosis we refer the reader to more specialized reviews on the meiotic SAC for more detailed information (Jones and Lane, 2013; Gorbsky, 2015; Touati and Wassmann, 2016). But the fact that SAC proteins are present in oocytes of organisms that seem not to be able to mount a detectable checkpoint delay in anaphase I onset is maybe a hint that additional, essential roles in meiosis have put 
evolutionary pressure on maintaining SAC proteins expressed in oocytes.

\section{KINASES IN DETAIL}

\section{Distinct Requirements for Bub1 Kinase Activity to Protect Centromeric Cohesin in Yeast and Mammals?}

The S/T kinase Bub1 is an essential conserved SAC component in mitosis and meiosis. Interestingly, the kinase activity of Bub1 is not required for checkpoint arrest in mouse oocytes (McGuinness et al., 2009; Vleugel et al., 2015; El Yakoubi et al., 2017), as in both $S$. cerevisiae and S. pombe mitosis (Vaur et al., 2005; Fernius and Hardwick, 2007). Like other SAC proteins, Bub1 protein localizes dynamically to unattached kinetochores through interaction with Bub3. Its main role in the SAC is the recruitment of Bub3-BubR1 and Cdc20 to unattached kinetochores. Contrary to the closely related BubR1 pseudokinase, Bub1 does not get incorporated into the MCC. Its role in the SAC is limited to promoting Bub3 binding to the kinetochore scaffold Spc105/Spc7/Knl1 that is phosphorylated by Mps1 in the absence of attachments. Bubl's role is thus essential for the recognition of the signal generated upon missing attachments (reviewed in Musacchio, 2015; Sacristan and Kops, 2015).

\section{Bub1 in Yeast Meiosis}

The first indication that Bub1 may play a role in meiosis that is distinct from its checkpoint function came from a study in S. pombe (Bernard et al., 2001). In contrast to mad2 $\Delta$ cells, bub $1 \Delta$ cells were found to prematurely separate sister chromatids and the meiotic cohesin subunit, Rec8, was removed from centromeres concomitant with arm cohesin during meiosis I (Bernard et al., 2001). This indicated that Bub1 is important for the protection of cohesion at centromeres in this organism. At least in $S$. pombe, the kinase activity of Bubl was found to be important for localization of Sgol during meiosis (Kawashima et al., 2010). An explanation for these findings came with the discovery of shugoshins in both $S$. pombe and S. cerevisiae (Katis et al., 2004; Kitajima et al., 2004; Marston et al., 2004; Rabitsch et al., 2004). Shugoshins, along with protein phosphatase 2A, shield pericentromeric cohesin from separase during the first meiotic division (Brar et al., 2006; Kitajima et al., 2006; Riedel et al., 2006; Ishiguro et al., 2010; Katis et al., 2010). To achieve this specific protection, shugoshins must be localized to the pericentromeric region, and this is the key non-SAC role of Bub1 in both S. pombe and S. cerevisiae meiosis (Kitajima et al., 2004; Kiburz et al., 2005; Riedel et al., 2006). Bub1, itself localized on the kinetochore, phosphorylates histone $\mathrm{H} 2 \mathrm{~A}$ at a specific residue ( $\mathrm{S} 121$ in yeasts) to create a binding site on the nucleosome for shugoshin (Sgo1). Later work has established an important role for this modification, and shugoshin also in mitotic cells (reviewed in Marston, 2015). During meiosis II in S. cerevisiae, however, Bub1 does not need to be retained at kinetochores for persistence of pericentromeric shugoshin-PP2A (ArgüelloMiranda et al., 2017). Instead evidence suggests that Mps1 is the critical kinase in localizing shugoshin-PP2A during meiosis II in budding yeast Argüello-Miranda et al., 2017; see below).

\section{Bub1 in Oocytes}

Complete loss of Bub1 in oocytes leads to gross missegregations, strong acceleration of prometaphase I and sterility (McGuinness et al., 2009). Importantly, SAC control can be rescued by expressing a kinase-dead version of Bub1 in oocytes, showing that also in oocytes, the kinase activity of Bub1 is not required for SAC signaling (McGuinness et al., 2009).

In addition to its role in the SAC, Bubl kinase activity was shown to be required for chromosome congression in somatic cells (Ricke et al., 2012; Asghar et al., 2015). Rescue experiments of Bub1-conditional knock-out oocytes indicate that the kinase domain of Bub1 plays only a minor role for chromosome congression in oocytes (McGuinness et al., 2009). Loss of Bub1 kinase activity in oocytes conditionally expressing only a kinasedead version of Bubl results in an arrest in metaphase I, or anaphase I onset with a significant delay due to checkpoint activation, when these oocytes are cultured in vitro (El Yakoubi et al., 2017). In vivo matured oocytes devoid of Bubl kinase activity eventually exit metaphase I arrest and progress into meiosis II where they can be fertilized. Bub1 kinase-dead mice are therefore fertile (Ricke et al., 2012). This result indicates that even though chromosome congression can in part be rescued by expressing Bub1 not harbouring any kinase activity, Bub1 protein still plays a role in stable attachment and alignment of chromosomes, and in its absence, the SAC is activated (El Yakoubi et al., 2017).

Besides its canonical role in the checkpoint and its role for chromosome congression, mammalian Bub1 generates a histone mark by phosphorylating T120 (human), or T121 (mouse) of $\mathrm{H} 2 \mathrm{~A}$, in somatic and germ cells, similar to yeast. In mammalian mitosis this histone mark provides a direct binding site for Sgo1 at the kinetochore (Kawashima et al., 2010; Liu et al., 2015) and is required for focused localization of Sgol at the centromere, as in yeast (Tang et al., 2004; Kitajima et al., 2005; Kawashima et al., 2010; Liu et al., 2013b). Sgo1 is important during mitosis to protect centromeric cohesin from the nonproteolytic pathway of cohesin removal that occurs in mitotic prophase, at the hands of Wapl (Hauf et al., 2005; Kueng et al., 2006; Shintomi and Hirano, 2009; Liu et al., 2013b). However, in human mitotic cells, this protective function of Sgol is provided by a pool associated with cohesin in the inner centromere, rather than the Bub1 kinase-dependent $\mathrm{H} 2 \mathrm{~A}$-bound kinetochoreassociated pool. Bub1-dependent binding of Sgol to H2A does, however, play an indirect role in cohesin protection because it is a prerequisite for the transcription-dependent translocation to cohesin to the inner centromere (Liu et al., 2013a,b, 2015). By analogy with mitosis, it was assumed that the same histone mark would contribute to the protection of centromeric cohesin in meiosis, but this time to protect cohesin from cleavage by separase. Surprisingly though, the kinase activity of Bub1 and phosphorylation of H2A T121 are not required for Sgo2 localization and protection of centromeric cohesin in oocytes, even though Bub1 itself, independently of its kinase activity, contributes to meiotic Sgo2 localization and cohesin protection 
(McGuinness et al., 2009; El Yakoubi et al., 2017). Accordingly, mice harbouring only kinase-dead Bub1 are fertile, and oocytes do not missegregate sister chromatids. In contrast, complete loss of Bub1 in oocytes leads to some precocious sister chromatid segregation, in agreement with the fact that Bub1 protein independently of its kinase activity and phosphorylation of $\mathrm{H} 2 \mathrm{~A}$ participates in the centromeric recruitment of Sgo2 (McGuinness et al., 2009; El Yakoubi et al., 2017), Figure 3.

\section{Summary}

While the importance of Bub1 protein in both mitotic and meiotic divisions across species is clear, the requirement of its kinase activity differs. There is a general role for Bubl kinases in phosphorylating Histone $\mathrm{H} 2 \mathrm{~A}$ to create a direct binding site for shugoshins. However, the importance of this Bub1 kinasedependent histone H2A-bound shugoshin pool for cohesin protection in meiosis differs, being dispensible in mouse oocytes and required only during meiosis I, in S. cerevisiae (Table 1).

\section{Mps1: Regulating Kinetochore-Microtubule Attachments and the Cohesin Protector}

Mps1, which can be viewed as the most upstream component of the SAC, also plays a central role in other aspects of mitosis and meiosis (Liu and Winey, 2012; Ciliberto and Hauf, 2017). S. cerevisiae MPS1 was originally identified as being important for the duplication of the yeast centrosome/spindle pole body (Winey et al., 1991) but later found to also function in the SAC (Weiss and Winey, 1996). The S. pombe Mps1 homolog, Mph1,

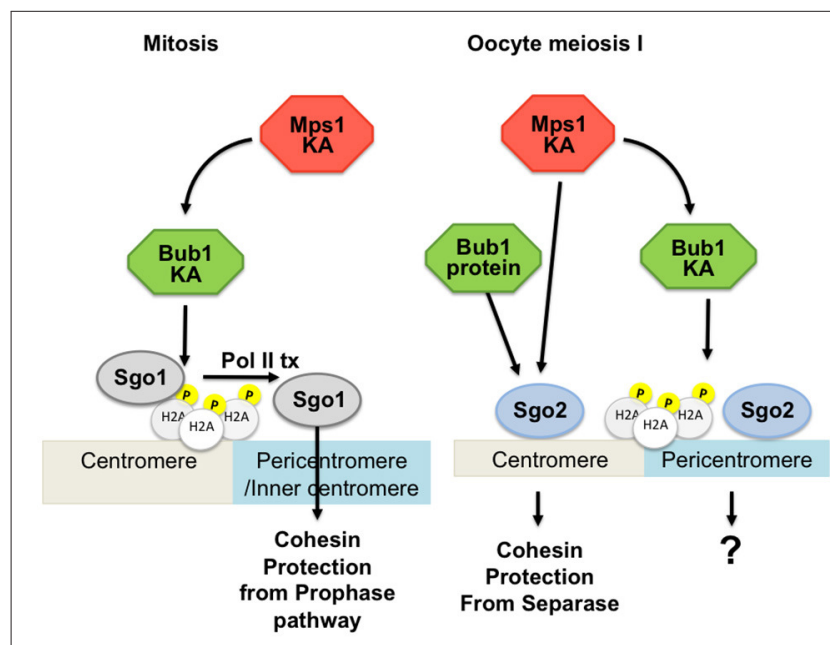

FIGURE 3 | Involvement of Bub1 and Mps1 kinases in Sgo2 localization in mouse oocytes. On the left: Sgo1 in mitosis is localized by Bub1-dependent phosphorylation of $\mathrm{H} 2 \mathrm{~A}$ and transcription for protection of cohesin. On the right: Two pools of Sgo2 can be distinguished in oocyte meiosis I, one of which is localized independently of $\mathrm{H} 2 \mathrm{~A}$ phosphorylation by Bub1, and which is required for protection of centromeric cohesin. Mps1 kinase is involved in localizing the pool of Sgo2 required for protection, at the centromere. Bub1 kinase is required for localizing a pool of $\mathrm{Sgo} 2$ in an $\mathrm{H} 2 \mathrm{~A}$-phosphorylation dependent manner, but this pool is largely dispensible for protection of centromeric cohesin in meiosis I. KA, kinase activity; Pol II tx, Polymerase II dependent transcription. is also critical for the SAC, but unlike S. cerevisiae Mps1, Mph1 is not required for SPB duplication, and therefore $M P H 1$ is not an essential gene (He et al., 1998). This kinase plays a conserved and key role in the SAC, phosphorylating MELT repeats in the outer kinetochore scaffold protein Spc105/Spc7/Knl1, which serves as a platform for other SAC proteins to generate the MCC (see Musacchio, 2015; Sacristan and Kops, 2015 for reviews). In some organisms, Mps1 has been shown to be required together with Aurora B kinase for error correction of incorrect microtubulekinetochore attachments and for the centromeric localization of Sgo1 (van der Waal et al., 2012; Williams et al., 2016), which protects centromeric cohesin from Wapl-dependent cohesin removal in mammalian mitotic prophase. However, Mps1 is not essential for localization of the other shugoshin paralog, Sgo2 in mammals, which does not contribute to mitotic cohesin protection (van der Waal et al., 2012).

\section{Mps1 in Yeast Meiosis}

As in mitosis, S. cerevisiae Mps1 is essential for spindle pole body duplication and chromosome segregation during meiosis (Straight et al., 2000). Hypomorphic mps1 mutations that support vegetative growth have catastrophic effects on meiosis, indicating that meiosis is especially reliant on Mps1 (Straight et al., 2000; Meyer et al., 2013). Elegant live cell imaging in meiotic cells showed that one critical role of Mps1 in chromosome segregation during meiosis is to generate force-bearing attachments of kinetochores to microtubules (Meyer et al., 2013). The behavior of chromosomes in hypomorphic mps1 mutants in meiosis indicates that Mps1 achieves this by converting the attachment of kinetochores to the sides (lateral) to the ends (end-on) of microtubules.

In addition, although anchor-away or kinase inhibition experiments revealed that $S$. cerevisiae Mps1 is dispensable for pericentromeric cohesin protection during meiosis I, conversely, during meiosis II it becomes important for this process, through maintaining the localization of shugoshin-PP2A, as described above (Argüello-Miranda et al., 2017). Indeed, APC/C $\mathrm{C}^{\mathrm{Cd} 20}$ dependent degradation of both shugoshin-PP2A and Mps1 is required for chromosome segregation at meiosis II, indicating that inactivation of Mps1 contributes to the deprotection of pericentromeric cohesin during meiosis II (Jonak et al., 2017). These findings are suggestive of a hand-off between Bub1 and Mps1 in cohesin protection, with the former kinase localizing Sgol during meiosis I and the latter kinase localizing Sgol during meiosis II. Whether this is a strict division of labour, or if there is interplay between these kinases during meiosis, as is well established during mitosis (London et al., 2012; Shepperd et al., 2012; Yamagishi et al., 2012), is not currently clear. Similarly, the relevant target of Mps1 and the requirement of H2A-S121P during meiosis II remain unknown.

\section{Mps1 in Mouse Oocytes}

In mouse oocytes, Mps1 kinase activity is essential for proper chromosome segregation (Touati et al., 2015). Anaphase I onset takes place before chromosomes have had time to congress to the metaphase plate, and gross missegregations lead to the generation of aneuploid oocytes that cannot give rise to viable embryos 


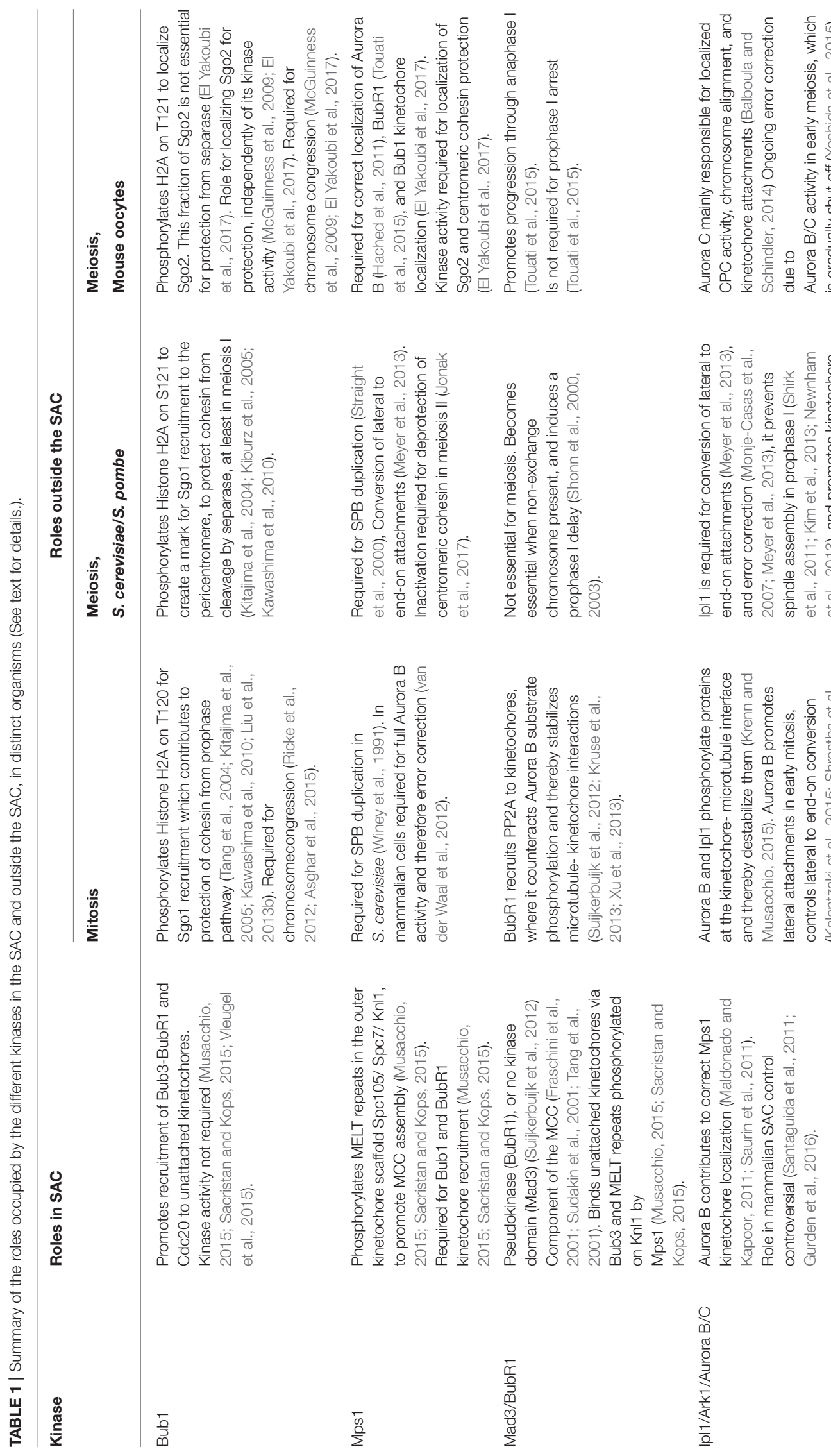


after fertilization. Without fully functional Mps1, the SAC is abolished: Mad2 is not recruited to unattached kinetochores, and the $\mathrm{APC} / \mathrm{C}$ is activated precociously. Interestingly, even though anaphase I onset is strongly accelerated, oocytes still establish stable end-on kinetochore-microtubule attachments in the absence of functional Mps1, indicating that Mps1 is not required for initial attachments (Hached et al., 2011; Touati et al., 2015). Preventing anaphase I onset allows oocytes to congress chromosomes to the metaphase plate, except those that are close to the poles, which seem to remain stably attached to one pole without being able to establish end-on attachments coming from the other pole (Hached et al., 2011). Even when accelerated anaphase I onset is prohibited, oocytes cannot establish correct attachments of bivalents at the poles, indicating that error correction is not properly functional in oocytes without Mps1. Indeed, Aurora B kinase, which is required for severing wrongly attached microtubules, is mis-localized in oocytes not harboring fully functional Mps1, and Mps1 localization to the kinetochore is decreased without Aurora B kinase activity (Hached et al., 2011; El Yakoubi et al., 2017).

Apart from Mps1's well established role in checkpoint control, Mps1 kinase activity in oocytes is essential for correct Sgo2 localization. In the absence of Mps1 kinase activity, Sgo2 recruitment to the centromere is reduced in meiosis I. This leads to failures in proper centromeric cohesin protection and the separation of some sister chromatids at the first meiotic division. Importantly, this recruitment of Sgo2 occurs independently of the canonical Histone H2A-T120 mark, and does not require kinetochore localization of Mps1 (Figure 3). Hence, oocytes devoid of correctly localized Mps1 but still harboring Mps1 kinase activity, are defective in checkpoint control, but not cohesin protection (El Yakoubi et al., 2017). The molecular targets of Mps1 kinase for Sgo2 localization at the centromere and for cohesin protection in meiosis I are still unknown.

\section{Summary}

Mps1 is required for spindle pole duplication in yeast meiosis, but not for centrosome duplication in mammalian oocytes, which divide without centrosomes. Cohesin protection in the first meiotic division in mouse oocytes depends on Mps1 kinasedependent localization of Sgo2 to the centromere, whereas in S. cerevisiae, Mps1 kinase is dispensable for cohesin protection, but required for sister chromatid segregation in meiosis II. Mps1's role for meiosis II in oocytes is still unknown.

\section{Mad3/BubR1: Not Requiring Any Kinase Activity}

Mad3 in yeast and its mammalian counterpart, BubR1, are both components of the MCC, with homology to Bub1 (see (Musacchio, 2015; Sacristan and Kops, 2015) for reviews). Unlike mammalian BubR1, yeast Mad3 lacks a kinase domain. Mammalian BubR1 is an untypical pseudo-kinase, which is thought to have maintained conserved residues for a kinase domain throughout evolution for structural reasons (Suijkerbuijk et al., 2012). Catalytic activity of BubR1 is not required for any of its functions, at least in mammalian cells. BubR1 is a component of the MCC and therefore essential for the spindle checkpoint.
It binds to unattached kinetochores through interaction with Bub3, which is recruited to phosphorylated MELT repeats of Knl1 by the Bub3-Bub1 complex. The MCC is then thought to be formed at the unattached kinetochore with simultaneously recruited Cdc20 and Mad2.

\section{Mad3 in Yeasts}

Unlike Mad2 or Mad1, Mad3 does not appear to be essential for meiosis in S. cerevisiae as cells lacking MAD3 exhibit normal spore viability (Shonn et al., 2000, 2003). Cells lacking MAD3 fail to activate the SAC in response to kinetochore-microtubule attachment defects in the same way as cells lacking MAD2, however, Mad3 does not seem to share the additional roles of Mad2 in orienting homologs during meiosis I (Shonn et al., 2003). Interestingly, Mad3 becomes critical in cells carrying a single chromosome that does not form crossovers and therefore does not generate tension during meiosis I. This non-exchange chromosome relies on a Mad3-mediated prophase delay that occurs during every meiosis for their proper segregation (Cheslock et al., 2005). Therefore current evidence supports the idea that the primary function of Mad3 in S. cerevisiae meiosis is to prolong prophase, as part of the SAC. Interestingly, human BubR1 was able to compensate for this function of Mad3 in S. cerevisiae meiosis (Cheslock et al., 2005).

\section{BubR1 in Mouse Oocytes}

Given the importance for BubR1 in mitotic SAC control it is not surprising that it is equally essential for meiotic SAC control. As for other SAC components, its loss of function leads to accelerated meiosis I in oocytes, with such high mis-segregation events that mice with a conditional, oocyte -specific knock-out of BubR1 are sterile. Importantly though, BubR1 has a role in promoting progression through anaphase I, independently of its SAC-related role. Oocytes devoid of BubR1 undergo accelerated anaphase I onset, degrade cyclin B1 and securin, and are then delayed in chromosome segregation and polar body extrusion (Touati et al., 2015). It is unknown whether this additional role of BubR1 is restricted to oocyte meiosis, or is equally promoting anaphase onset in mitosis. As BubR1's role for promoting progression through anaphase I is not essential and oocytes eventually manage to exit meiosis I, this function of BubR1, if conserved, may have been missed in somatic cells, due to the much more rapid progression through mitosis, compared to oocyte meiosis.

In oocytes, BubR1 was proposed to be required for the extended prophase arrest before entry into the first meiotic division (Homer et al., 2009), but this was not confirmed with a knock-out approach and is therefore most probably due to the morpholino-knock-down technique that has been used, without simultaneous assessment of knock-down levels in individual cells. Oocytes completely devoid of BubR1 remain correctly arrested in prophase I and upon release, enter meiosis I on time, indicating that BubR1 is not required for prophase I arrest (Touati et al., 2015).

The mitotic role of BubR1 in stabilizing kinetochoremicrotubule attachments is conserved in oocytes. But contrary to mitosis, BubR1 does not need to be localized to kinetochores 
to stabilize microtubules in oocytes (Touati et al., 2015). In mitosis, BubR1 recruits PP2A-B56 phosphatase to kinetochores, where it counteracts the microtubule-destabilizing activity of Aurora B (Suijkerbuijk et al., 2012; Kruse et al., 2013; Xu et al., 2013). In oocytes devoid of BubR1, stabilization of kinetochore fibers cannot be rescued by inhibiting Aurora B kinase activity, indicating that BubR1 promotes stabilization of kinetochore fibers in oocytes in a distinct manner, in the cytosol (Touati et al., 2015).

\section{Summary}

Both yeast Mad3 and mammalian BubR1 are components of the MCC. In S. cerevisiae meiosis, Mad3 is required for the segregation of non-exchange chromosomes by delaying prophase I. Cytosolic BubR1 in oocytes is necessary to stabilize kinetochore-microtubule interactions. BubR1 furthermore promotes progression through anaphase I.

\section{Ipl1/Ark1/Aurora B Is Important for Multiple Steps in Meiosis}

In mitosis, the role of the Aurora B kinase in the SAC signaling cascade is still controversial and beyond the scope of this review. As mentioned above, Aurora B is at least in part required for full Mps1 activity and thereby for prolonged maintenance of SAC arrest. Being an essential component of the chromosomal passenger complex (CPC), Aurora B destabilizes erroneous kinetochore-microtubule attachments that are not under tension from the bipolar spindle, to permit establishment of correct attachments, and controls the conversion of lateral to end-on attachments in mitosis (Kalantzaki et al., 2015; Shrestha et al., 2017), for review see (van der Horst and Lens, 2014; Krenn and Musacchio, 2015). Independent of its potential role in SAC signaling, Aurora B maintains SAC activity by creating unattached kinetochores. Besides error correction, Aurora B is important for chromosome condensation and cytokinesis in mitosis (for review see van der Horst and Lens, 2014).

\section{Ipl1/Ark1 in Yeast}

Budding yeast Aurora B kinase has been implicated in many processes that are essential for successful meiosis. Meiosisspecific depletion of budding yeast Aurora B kinase, Ipl1, revealed that it is required for the biorientation of both homologs in meiosis I and sister chromatids during meiosis II, suggesting that it is important for error correction in both meiotic divisions in addition to mitosis (Monje-Casas et al., 2007). This was later confirmed through live cell imaging studies (Meyer et al., 2013). Ipl1 is also important earlier, during meiotic prophase, where it is important for coordination of meiotic processes to prevent premature chromosome segregation (Kim et al., 2013). Several studies observed an uncoupling of meiotic events in Ipl1-depleted cells (Jordan et al., 2009; Shirk et al., 2011; Kim et al., 2013; Newnham et al., 2013). Ipl1 was originally thought to be important for timely disassembly of the normally prophase-specific synaptonemal complex (SC) since SCs were found to co-exist with the meiosis I spindle, which should form only upon prophase exit, in Ipl1-depleted cells (Jordan et al., 2009). However, later studies showed that this phenotype was largely due to assembly of the meiosis I spindle already in prophase (Shirk et al., 2011; Kim et al., 2013; Newnham et al., 2013). How Ipl1 prevents spindle assembly in prophase remains unclear. Ipl1 localizes on microtubules next to the yeast centrosome (called the spindle pole body) and dissociation of Ipl1 at prophase I exit occurs coincident with spindle assembly (Kim et al., 2013). Therefore, one possibility is that Ipl1 acts to destabilize microtubules near SPBs in a similar manner to disruption of kinetochore microtubules during error correction (Kim et al., 2013). A further function of Ipl1, which also acts to prevent premature chromosome segregation, is to trigger the shedding of the outer kinetochore during meiotic prophase, thereby abolishing the ability of kinetochores to interact with microtubules and promoting assembly of the monopolin complex, which directs kinetochore co-orientation during meiosis I (Kim et al., 2013; Meyer et al., 2015). In addition to its microtubule/kinetochore-related functions, Ipl1 is important for the maintenance of centromeric cohesion, potentially due to a failure to maintain the protective Sgo1PP2A complex at centromeres (Monje-Casas et al., 2007; Yu and Koshland, 2007).

S. pombe Aurora B kinase, Ark1, also plays multiple essential roles that impact meiotic chromosome segregation. In addition to promoting homolog biorientation, Ark1 is essential for sister kinetochore co-orientation in this organism (Hauf et al., 2007). Interestingly, loading of Ark1 to kinetochores relies on S. pombe shugoshin 2 (Sgo2), which is distinct from shugoshin 1 (Sgo1) that protects cohesion in this organism (Kawashima et al., 2007). Ark1 suppresses the attachment of microtubules from opposite poles to binding sites on the same kinetochore (merotelic attachment) during meiosis $I$, in a manner dependent on the presence of chiasmata (Sakuno et al., 2011). During this chiasmadependent realignment process, Ark1 relocates to the inner centromere on the inside face of the bivalent, away from the site of microtubule attachment (Sakuno et al., 2011). This suggests that Ark 1 is responsive to tension across homologs, but the molecular mechanism of how this tension is sensed remains completely unknown.

\section{Aurora B in Mouse Oocytes}

Mammalian oocytes harbor Aurora B and the closely related Aurora C kinase (Sasai et al., 2004), which is lacking certain destruction motifs found in Aurora B, and is therefore more stably expressed (Schindler et al., 2012). Oocyte-specific Aurora C knock-out only leads to subfertility, showing that Aurora $\mathrm{C}$ is important for meiotic maturation, but not essential to generate oocytes that can be fertilized (Schindler et al., 2012), suggesting a certain redundancy in the roles of these two kinases. It is mainly Aurora C that promotes localized CPC activity, chromosome alignment and establishment of kinetochore attachments, required for efficient meiotic progression (Balboula and Schindler, 2014), probably due to higher protein stability compared to Aurora B.

Studies on error correction in meiosis have used small molecule inhibitors that do not allow to distinguish between the respective roles of Aurora $\mathrm{B}$ and $\mathrm{C}$, but Aurora B/C kinase-dependent error correction was shown to take place in 
prometaphase I, when attachments undergo multiple attachment cycles (Kitajima et al., 2011; Yoshida et al., 2015). Aurora $\mathrm{B} / \mathrm{C}$ activity continuously decreases as oocytes progress into metaphase I. Artificially down-regulating Aurora B/C during meiotic maturation was shown to help oocytes establish endon attachments and prevent aneuploidies (Yoshida et al., 2015). Nevertheless, this does not mean that Aurora B/C's role in meiosis is expendable or even harmful for establishing correct attachments in general, as attested by expressing a dominant negative version of Aurora $\mathrm{C}$ in oocytes (Balboula and Schindler, 2014). Also, the importance of Aurora B/C to detect attachments that are not under tension before metaphase-to-anaphase transition has not yet been addressed in oocytes.

Maybe in agreement with a higher stability of Aurora C, the kinase is not only detected in the centromere region but also at the axis in between the sister chromatids, in meiosis I. Aurora $\mathrm{C}$ as part of the $\mathrm{CPC}$ is recruited by the Haspindependent phospho-Histone $\mathrm{H} 3$ mark to the centromere region and interchromatid axis in meiosis I (Nguyen et al., 2014). Aurora C's role at the interchromatid axis is still unknown, but may be related to its role in chromosome condensation (Nguyen et al., 2014).

\section{Summary}

Ipl1, Ark1, and Aurora B/C fulfill multiple roles in meiosis, such as for chromosome condensation, preventing premature chromosome segregation, and cytokinesis. The kinase counteracts the establishment of stable, end-on attachments of kinetochores to microtubules, probably in all organisms as part of error correction of tension-less attachments.

\section{The Role of SAC Kinases in CSF Arrest}

It was proposed that in addition to Mad1 and Mad2 (Tunquist et al., 2003), the SAC kinase Bub1 participates in establishing CSF arrest by down-regulating Cyclin E-Cdk2, in Xenopus laevis oocytes, with the caveat that these experiments were performed using overexpressed proteins (Tunquist et al., 2002). A role in CSF arrest for endogenous SAC kinases has not been shown. There is enough evidence in mammalian oocytes excluding an essential role for SAC kinases in CSF arrest, both using conditional knock-out mouse models and expression of

\section{REFERENCES}

Argüello-Miranda, O., Zagoriy, I., Mengoli, V., Rojas, J., Jonak, K., Oz, T., et al. (2017). Casein kinase 1 coordinates cohesin cleavage, gametogenesis, and exit from $\mathrm{M}$ phase in meiosis II. Dev. Cell 40, 37-52. doi: 10.1016/j.devcel.2016.11.021

Asghar, A., Lajeunesse, A., Dulla, K., Combes, G., Thebault, P., Nigg, E. A., et al. (2015). Bubl autophosphorylation feeds back to regulate kinetochore docking and promote localized substrate phosphorylation. Nat. Commun. 6:8364. doi: $10.1038 /$ ncomms 9364

Bähler, J., Schuchert, P., Grimm, C., and Kohli, J. (1991). Synchronized meiosis and recombination in fission yeast: observations with pat1114 diploid cells. Curr. Genet. 19, 445-451. doi: 10.1007/BF003 12735 dominant negative constructs (Tsurumi et al., 2004; Touati et al., 2015). This indicates that beyond potential other functions in meiosis II, such as SAC control, Sgo2 localization, and stabilization of kinetochore-microtubule interactions, SAC kinases are not implicated in establishing or maintaining a CSF arrest. Future work will show whether SAC kinases have additional, as yet unknown, roles in meiosis II.

\section{CONCLUSION}

The specificities of the meiotic cell division require adaptation of known regulatory mechanisms that govern somatic cell divisions. Kinases that are important for mitotic SAC control fulfill important additional roles in meiosis, independent of a functional SAC (see Table 1 for a summary of the different roles SAC kinases play in meiosis). Well-known model organisms such as mouse, yeast and Drosophila, emerging model systems, and comparative evolutionary studies will help us to obtain a better picture of the multiple steps regulated by these kinases. Importantly, even though the result of meiosis is the same in all models (the generation of haploid cells), details in the molecular pathways ensuring the correct segregation of chromosomes and sister chromatids may vary. These differences provide key insight that will help us understand the essential parts and targets of each pathway for the generation of euploid gametes.

\section{AUTHOR CONTRIBUTIONS}

KW has written most of the part of this review dealing with meiosis in higher organisms, and AM has written most of the part on meiosis in yeast. Both authors have corrected the whole manuscript.

\section{FUNDING}

KW acknowledges financial support through grants by the Agence Nationale de la Recherche (ANR-16-CE92-0007-01), a grant "Equipe FRM" by the Fondation de la Recherche Médicale (Equipe DEQ20160334921), and core funding by UPMC and CNRS. AM gratefully acknowledges support from Wellcome through a Senior Research Fellowship (107827) and core funding for the Wellcome Centre Cell Biology (203149).
Balboula, A. Z., and Schindler, K. (2014). Selective disruption of aurora C kinase reveals distinct functions from aurora B kinase during meiosis in mouse oocytes. PLoS Genet. 10:e1004194. doi: 10.1371/journal.pgen.1004194

Batiha, O., and Swan, A. (2012). Evidence that the spindle assembly checkpoint does not regulate APC(Fzy) activity in Drosophila female meiosis. Genome 55, 63-67. doi: 10.1139/g11-079

Bernard, P., Maure, J. F., and Javerzat, J. P. (2001). Fission yeast Bubl is essential in setting up the meiotic pattern of chromosome segregation. Nat. Cell Biol. 3, 522-526. doi: 10.1038/35074598

Brar, G. A., Kiburz, B. M., Zhang, Y., Kim, J. E., White, F., and Amon, A. (2006). Rec8 phosphorylation and recombination promote the step-wise loss of cohesins in meiosis. Nature 441, 532-536. doi: 10.1038/nature04794

Buffin, E., Emre, D., and Karess, R. E. (2007). Flies without a spindle checkpoint. Nat. Cell Biol. 9, 565-572. doi: 10.1038/ncb1570 
Carlile, T. M., and Amon, A. (2008). Meiosis I is established through division-specific translational control of a cyclin. Cell 133, 280-291. doi: 10.1016/j.cell.2008.02.032

Cheslock, P. S., Kemp, B. J., Boumil, R. M., and Dawson, D. S. (2005). The roles of MAD1, MAD2 and MAD3 in meiotic progression and the segregation of nonexchange chromosomes. Nat. Genet. 37, 756-760. doi: 10.1038/ ng1588

Chia, M., and van Werven, F. J. (2016). Temporal expression of a master regulator drives synchronous sporulation in budding yeast. G3 6, 3553-3560. doi: $10.1534 / \mathrm{g} 3.116 .034983$

Chiang, T., Duncan, F. E., Schindler, K., Schultz, R. M., and Lampson, M. A. (2010). Evidence that weakened centromere cohesion is a leading cause of age-related aneuploidy in oocytes. Curr. Biol. 20, 1522-1528. doi: 10.1016/j.cub.2010.06.069

Ciliberto, A., and Hauf, S. (2017). Cell cycle: micromanaging checkpoint proteins. Elife 6:e25001. doi: 10.7554/eLife.25001

Cipak, L., Hyppa, R. W., Smith, G. R., and Gregan, J. (2012). ATP analog-sensitive Pat1 protein kinase for synchronous fission yeast meiosis at physiological temperature. Cell Cycle 11, 1626-1633. doi: 10.4161/cc.20052

Collin, P., Nashchekina, O., Walker, R., and Pines, J. (2013). The spindle assembly checkpoint works like a rheostat rather than a toggle switch. Nat. Cell Biol. 15, 1378-1385. doi: 10.1038/ncb2855

Dobles, M., Liberal, V., Scott, M. L., Benezra, R., and Sorger, P. K. (2000). Chromosome missegregation and apoptosis in mice lacking the mitotic checkpoint protein Mad2. Cell 101, 635-645. doi: 10.1016/S0092-8674(00)80875-2

El Yakoubi, W., Buffin, E., Cladière, D., Gryaznova, Y., Berenguer, I., Touati, S. A., et al. (2017). Mps1 kinase-dependent Sgo2 centromere localisation mediates cohesin protection in mouse oocyte meiosis I. Nat. Commun. 8:694. doi: 10.1038/s41467-017-00774-3

El Yakoubi, W., and Wassmann, K. (2017). Meiotic divisions: no place for gender equality. Adv. Exp. Med. Biol. 1002, 1-17. doi: 10.1007/978-3-319-57127-0_1

Fernius, J., and Hardwick, K. G. (2007). Bubl kinase targets Sgol to ensure efficient chromosome biorientation in budding yeast mitosis. PLoS Genet. 3:e213. doi: 10.1371/journal.pgen.0030213

Fraschini, R., Beretta, A., Sironi, L., Musacchio, A., Lucchini, G., and Piatti, S. (2001). Bub3 interaction with Mad2, Mad3 and Cdc20 is mediated by WD40 repeats and does not require intact kinetochores. EMBO J. 20, 6648-6659. doi: $10.1093 / \mathrm{emboj} / 20.23 .6648$

Gilliland, W. D., Wayson, S. M., and Hawley, R. S. (2005). The meiotic defects of mutants in the Drosophila mps1 gene reveal a critical role of Mps1 in the segregation of achiasmate homologs. Curr. Biol. 15, 672-677. doi: 10.1016/j.cub.2005.02.062

Goldstein, L. S. (1981). Kinetochore structure and its role in chromosome orientation during the first meiotic division in male D. melanogaster. Cell 25, 591-602. doi: 10.1016/0092-8674(81)90167-7

Gorbsky, G. J. (2015). The spindle checkpoint and chromosome segregation in meiosis. FEBS J. 282, 2471-2487. doi: 10.1111/febs.13166

Gorbsky, G. J., Chen, R. H., and Murray, A. W. (1998). Microinjection of antibody to Mad2 protein into mammalian cells in mitosis induces premature anaphase. J. Cell Biol. 141, 1193-1205. doi: 10.1083/jcb.141.5.1193

Gui, L., and Homer, H. (2012). Spindle assembly checkpoint signalling is uncoupled from chromosomal position in mouse oocytes. Development, 139, 1941-1946. doi: 10.1242/dev.078352

Gurden, M. D., Anderhub, S. J., Faisal, A., and Linardopoulos, S. (2016). Aurora $B$ prevents premature removal of spindle assembly checkpoint proteins from the kinetochore: a key role for aurora B in mitosis. Oncotarget. [Epub ahead of print].

Hached, K., Xie, S. Z., Buffin, E., Cladiere, D., Rachez, C., Sacras, M., et al. (2011). Mps1 at kinetochores is essential for female mouse meiosis Development 138, 2261-2271. doi: 10.1242/dev.061317

Hassold, T., and Hunt, P. (2001). To err (meiotically) is human: the genesis of human aneuploidy. Nat. Rev. Genet. 2, 280-291. doi: 10.1038/35066065

Hassold, T., and Hunt, P. (2009). Maternal age and chromosomally abnormal pregnancies: what we know and what we wish we knew. Curr. Opin. Pediatr. 21, 703-708. doi: 10.1097/MOP.0b013e328332c6ab

Hauf, S., Biswas, A., Langegger, M., Kawashima, S. A., Tsukahara, T., and Watanabe, Y. (2007). Aurora controls sister kinetochore mono-orientation and homolog bi-orientation in meiosis-I. EMBO J. 26, 4475-4486. doi: 10.1038/sj.emboj.7601880

Hauf, S., Roitinger, E., Koch, B., Dittrich, C. M., Mechtler, K., and Peters, J. M. (2005). Dissociation of cohesin from chromosome arms and loss of arm cohesion during early mitosis depends on phosphorylation of SA2. PLoS Biol. 3:e69. doi: 10.1371/journal.pbio.0030069

He, E., Kapuy, O., Oliveira, R. A., Uhlmann, F., Tyson, J. J., and Novak, B. (2011). System-level feedbacks make the anaphase switch irreversible. Proc. Natl. Acad. Sci. U.S.A. 108, 10016-10021. doi: 10.1073/pnas.1102106108

He, X., Jones, M. H., Winey, M., and Sazer, S. (1998). Mph1, a member of the Mps1-like family of dual specificity protein kinases, is required for the spindle checkpoint in S. pombe. J. Cell Sci. 111(Pt 12), 1635-1647.

Herbert, M., Levasseur, M., Homer, H., Yallop, K., Murdoch, A., and McDougall, A. (2003). Homologue disjunction in mouse oocytes requires proteolysis of securin and cyclin B1. Nat. Cell Biol. 5, 1023-1025. doi: 10.1038/ncb1062

Homer, H. A., McDougall, A., Levasseur, M., Murdoch, A. P., and Herbert, M. (2005). Mad2 is required for inhibiting securin and cyclin B degradation following spindle depolymerisation in meiosis I mouse oocytes. Reproduction 130, 829-843. doi: 10.1530/rep.1.00856

Homer, H., Gui, L., and Carroll, J. (2009). A spindle assembly checkpoint protein functions in prophase I arrest and prometaphase progression. Science 326, 991-994. doi: 10.1126/science.1175326

Hoyt, M. A., Totis, L., and Roberts, B. T. (1991). S. cerevisiae genes required for cell cycle arrest in response to loss of microtubule function. Cell 66, 507-517.

Hunter, N. (2015). Meiotic recombination: the essence of heredity. Cold Spring Harb. Perspect. Biol. 7:a016618. doi: 10.1101/cshperspect.a016618

Ishiguro, T., Tanaka, K., Sakuno, T., and Watanabe, Y. (2010). Shugoshin-PP2A counteracts casein-kinase-1-dependent cleavage of Rec8 by separase. Nat. Cell Biol. 12, 500-506. doi: 10.1038/ncb2052

Jessberger, R. (2012). Age-related aneuploidy through cohesion exhaustion. EMBO Rep. 13, 539-546. doi: 10.1038/embor.2012.54

Jonak, K., Zagoriy, I., Oz, T., Graf, P., Rojas, J., Mengoli, V., et al. (2017). APC/C$\mathrm{Cdc} 20$ mediates deprotection of centromeric cohesin at meiosis II in yeast. Cell Cycle 16, 1145-1152. doi: 10.1080/15384101.2017.1320628

Jones, K. T., and Lane, S. I. (2013). Molecular causes of aneuploidy in mammalian eggs. Development 140, 3719-3730. doi: 10.1242/dev.090589

Jordan, P., Copsey, A., Newnham, L., Kolar, E., Lichten, M., and Hoffmann, E. (2009). Ipl1/Aurora B kinase coordinates synaptonemal complex disassembly with cell cycle progression and crossover formation in budding yeast meiosis. Genes Dev. 23, 2237-2251. doi: 10.1101/gad.536109

Kakui, Y., Sato, M., Tanaka, K., and Yamamoto, M. (2011). A novel fission yeast mei4 mutant that allows efficient synchronization of telomere dispersal and the first meiotic division. Yeast 28, 467-479. doi: 10.1002/yea.1851

Kalantzaki, M., Kitamura, E., Zhang, T., Mino, A., Novak, B., and Tanaka, T. U. (2015). Kinetochore-microtubule error correction is driven by differentially regulated interaction modes. Nat. Cell Biol. 17:530. doi: 10.1038/ncb3128

Kalitsis, P., Earle, E., Fowler, K. J., and Choo, K. H. (2000). Bub3 gene disruption in mice reveals essential mitotic spindle checkpoint function during early embryogenesis. Genes Dev. 14, 2277-2282. doi: 10.1101/gad.827500

Kamenz, J., and Hauf, S. (2014). Slow checkpoint activation kinetics as a safety device in anaphase. Curr. Biol. 24, 646-651. doi: 10.1016/j.cub.2014.02.005

Katis, V. L., Galova, M., Rabitsch, K. P., Gregan, J., and Nasmyth, K. (2004). Maintenance of cohesin at centromeres after meiosis I in budding yeast requires a kinetochore-associated protein related to MEI-S332. Curr. Biol. 14, 560-572. doi: 10.1016/j.cub.2004.03.001

Katis, V. L., Lipp, J. J., Imre, R., Bogdanova, A., Okaz, E., Habermann, B., et al. (2010). Rec8 phosphorylation by casein kinase 1 and Cdc7-Dbf4 kinase regulates cohesin cleavage by separase during meiosis. Dev. Cell. 18, 397-409. doi: 10.1016/j.devcel.2010.01.014

Kawashima, S. A., Tsukahara, T., Langegger, M., Hauf, S., Kitajima, T. S., and Watanabe, Y. (2007). Shugoshin enables tension-generating attachment of kinetochores by loading Aurora to centromeres. Genes Dev. 21, 420-435. doi: 10.1101/gad.1497307

Kawashima, S. A., Yamagishi, Y., Honda, T., Ishiguro, K., and Watanabe, Y. (2010). Phosphorylation of H2A by Bub1 prevents chromosomal instability through localizing shugoshin. Science 327, 172-177. doi: 10.1126/science.1180189

Khodjakov, A., and Pines, J. (2010). Centromere tension: a divisive issue. Nat. Cell Biol. 12, 919-923. doi: 10.1038/ncb1010-919 
Kiburz, B. M., Reynolds, D. B., Megee, P. C., Marston, A. L., Lee, B. H., Lee, T. I., et al. (2005). The core centromere and Sgol establish a 50-kb cohesin-protected domain around centromeres during meiosis I. Genes Dev. 19, 3017-3030. doi: $10.1101 /$ gad.1373005

Kim, S., Meyer, R., Chuong, H., and Dawson, D. S. (2013). Dual mechanisms prevent premature chromosome segregation during meiosis. Genes Dev. 27, 2139-2146. doi: 10.1101/gad.227454.113

Kitajima, T. S., Hauf, S., Ohsugi, M., Yamamoto, T., and Watanabe, Y. (2005). Human Bubl defines the persistent cohesion site along the mitotic chromosome by affecting Shugoshin localization. Curr. Biol. 15, 353-359. doi: 10.1016/j.cub.2004.12.044

Kitajima, T. S., Kawashima, S. A., and Watanabe, Y. (2004). The conserved kinetochore protein shugoshin protects centromeric cohesion during meiosis. Nature 427, 510-517. doi: 10.1038/nature02312

Kitajima, T. S., Ohsugi, M., and Ellenberg, J. (2011). Complete kinetochore tracking reveals error-prone homologous chromosome biorientation in mammalian oocytes. Cell 146, 568-581. doi: 10.1016/j.cell.2011. 07.031

Kitajima, T. S., Sakuno, T., Ishiguro, K., Iemura, S., Natsume, T., Kawashima, S. A., et al. (2006). Shugoshin collaborates with protein phosphatase $2 \mathrm{~A}$ to protect cohesin. Nature 441, 46-52. doi: 10.1038/nature04663

Krenn, V., and Musacchio, A. (2015). The aurora B kinase in chromosome bi-orientation and spindle checkpoint signaling. Front. Oncol. 5:225. doi: $10.3389 /$ fonc. 2015.00225

Kruse, T., Zhang, G., Larsen, M. S., Lischetti, T., Streicher, W., Kragh Nielsen, T., et al. (2013). Direct binding between BubR1 and B56-PP2A phosphatase complexes regulate mitotic progression. J. Cell Sci. 126(Pt 5), 1086-1092. doi: $10.1242 /$ jcs. 122481

Kudo, N. R., Wassmann, K., Anger, M., Schuh, M., Wirth, K. G., Xu, H., et al. (2006). Resolution of chiasmata in oocytes requires separase-mediated proteolysis. Cell 126, 135-146. doi: 10.1016/j.cell.2006.05.033

Kueng, S., Hegemann, B., Peters, B. H., Lipp, J. J., Schleiffer, A., Mechtler, K., et al. (2006). Wapl controls the dynamic association of cohesin with chromatin. Cell 127, 955-967. doi: 10.1016/j.cell.2006.09.040

Lacefield, S., and Murray, A. W. (2007). The spindle checkpoint rescues the meiotic segregation of chromosomes whose crossovers are far from the centromere. Nat. Genet. 39, 1273-1277. doi: 10.1038/ng2120

Lagirand-Cantaloube, J., Ciabrini, C., Charrasse, S., Ferrieres, A., Castro, A., Anahory, T., et al. (2017). Loss of centromere cohesion in aneuploid human oocytes correlates with decreased kinetochore localization of the sac proteins Bub1 and Bubr1. Sci. Rep. 7:44001. doi: 10.1038/srep44001

Lan, Z. J., Xu, X., and Cooney, A. J. (2004). Differential oocyte-specific expression of Cre recombinase activity in GDF-9-iCre, Zp3cre, and Msx2Cre transgenic mice. Biol. Reprod. 71, 1469-1474. doi: 10.1095/biolreprod.104. 031757

Lane, S. I., Yun, Y., and Jones, K. T. (2012). Timing of anaphase-promoting complex activation in mouse oocytes is predicted by microtubule-kinetochore attachment but not by bivalent alignment or tension. Development 139, 1947-1955. doi: 10.1242/dev.077040

Lee, B. H., Kiburz, B. M., and Amon, A. (2004). Spo13 maintains centromeric cohesion and kinetochore coorientation during meiosis I. Curr. Biol. 14, 2168-2182. doi: 10.1016/j.cub.2004.12.033

Lewandoski, M., Wassarman, K. M., and Martin, G. R. (1997). Zp3-cre, a transgenic mouse line for the activation or inactivation of loxP-flanked target genes specifically in the female germ line. Curr. Biol. 7, 148-151. doi: 10.1016/S0960-9822(06)00059-5

Li, R., and Murray, A. W. (1991). Feedback control of mitosis in budding yeast. Cell 66, 519-531. doi: 10.1016/0092-8674(81)90015-5

Li, X., and Dawe, R. K. (2009). Fused sister kinetochores initiate the reductional division in meiosis I. Nat. Cell Biol. 11, 1103-1108. doi: 10.1038/ncb1923

Lister, L. M., Kouznetsova, A., Hyslop, L. A., Kalleas, D., Pace, S. L., Barel, J. C., et al. (2010). Age-related meiotic segregation errors in Mammalian oocytes are preceded by depletion of cohesin and Sgo2. Curr. Biol. 20, 1511-1521. doi: 10.1016/j.cub.2010.08.023

Liu, D., Shao, H., Wang, H., and Liu, X. J. (2014). Meiosis I in Xenopus oocytes is not error-prone despite lacking spindle assembly checkpoint. Cell Cycle 13, 1602-1606. doi: $10.4161 / c c .28562$
Liu, H., Jia, L., and Yu, H. (2013a). Phospho-H2A and cohesin specify distinct tension-regulated Sgo1 pools at kinetochores and inner centromeres. Curr. Biol. 23, 1927-1933. doi: 10.1016/j.cub.2013.07.078

Liu, H., Qu, Q., Warrington, R., Rice, A., Cheng, N., and Yu, H. (2015). Mitotic transcription installs Sgol at centromeres to coordinate chromosome segregation. Mol. Cell 59, 426-436. doi: 10.1016/j.molcel.2015.06.018

Liu, H., Rankin, S., and Yu, H. (2013b). Phosphorylation-enabled binding of SGO1-PP2A to cohesin protects sororin and centromeric cohesion during mitosis. Nat. Cell Biol. 15, 40-49. doi: 10.1038/ncb2637

Liu, X., and Winey, M. (2012). The MPS1 family of protein kinases. Annu. Rev. Biochem. 81, 561-585. doi: 10.1146/annurev-biochem-061611-090435

London, N., Ceto, S., Ranish, J. A., and Biggins, S. (2012). Phosphoregulation of Spc105 by Mps1 and PP1 regulates Bub1 localization to kinetochores. Curr. Biol. 22, 900-906. doi: 10.1016/j.cub.2012.03.052

Maldonado, M., and Kapoor, T. M. (2011). Constitutive Mad1 targeting to kinetochores uncouples checkpoint signalling from chromosome biorientation. Nat. Cell Biol. 13, 475-482. doi: 10.1038/ncb2223

Malmanche, N., Owen, S., Gegick, S., Steffensen, S., Tomkiel, J. E., and Sunkel, C. E. (2007). Drosophila BubR1 is essential for meiotic sister-chromatid cohesion and maintenance of synaptonemal complex. Curr. Biol. 17, 1489-1497. doi: 10.1016/j.cub.2007.07.042

Marston, A. L. (2015). Shugoshins: tension-sensitive pericentromeric adaptors safeguarding chromosome segregation. Mol. Cell Biol. 35, 634-648. doi: 10.1128/MCB.01176-14

Marston, A. L., and Amon, A. (2004). Meiosis: cell-cycle controls shuffle and deal. Nat. Rev. Mol. Cell Biol. 5, 983-997. doi: 10.1038/nrm1526

Marston, A. L., Tham, W. H., Shah, H., and Amon, A. (2004). A genomewide screen identifies genes required for centromeric cohesion. Science 303, 1367-1370. doi: 10.1126/science.1094220

McGuinness, B. E., Anger, M., Kouznetsova, A., Gil-Bernabe, A. M., Helmhart, W., Kudo, N. R., et al. (2009). Regulation of APC/C activity in oocytes by a Bubl-dependent spindle assembly checkpoint. Curr. Biol. 19, 369-380. doi: 10.1016/j.cub.2009.01.064

Meraldi, P., Draviam, V. M., and Sorger, P. K. (2004). Timing and checkpoints in the regulation of mitotic progression. Dev. Cell 7, 45-60. doi: 10.1016/j.devcel.2004.06.006

Meyer, R. E., Chuong, H. H., Hild, M., Hansen, C. L., Kinter, M., and Dawson, D. S. (2015). Ipl1/Aurora-B is necessary for kinetochore restructuring in meiosis I in Saccharomyces cerevisiae. Mol. Biol. Cell 26, 2986-3000. doi: 10.1091/mbc.E15-01-0032

Meyer, R. E., Kim, S., Obeso, D., Straight, P. D., Winey, M., and Dawson, D. S. (2013). Mps1 and Ipl1/Aurora B act sequentially to correctly orient chromosomes on the meiotic spindle of budding yeast. Science 339, 1071-1074. doi: $10.1126 /$ science. 1232518

Michaelis, C., Ciosk, R., and Nasmyth, K. (1997). Cohesins: chromosomal proteins that prevent premature separation of sister chromatids. Cell 91, 35-45. doi: 10.1016/S0092-8674(01)80007-6

Monje-Casas, F., Prabhu, V. R., Lee, B. H., Boselli, M., and Amon, A. (2007). Kinetochore orientation during meiosis is controlled by Aurora B and the monopolin complex. Cell 128, 477-490. doi: 10.1016/j.cell.2006.12.040

Musacchio, A. (2015). The molecular biology of spindle assembly checkpoint signaling dynamics. Curr. Biol. 25, R1002-R1018. doi: 10.1016/j.cub.2015.08.051

Nasmyth, K. (2011). Cohesin: a catenase with separate entry and exit gates? Nat. Cell Biol. 13, 1170-1177. doi: 10.1038/ncb2349

Newnham, L., Jordan, P. W., Carballo, J. A., Newcombe, S., and Hoffmann, E. (2013). Ipl1/Aurora kinase suppresses S-CDK-driven spindle formation during prophase I to ensure chromosome integrity during meiosis. PLoS ONE 8:e83982. doi: 10.1371/journal.pone.0083982

Nguyen, A. L., Gentilello, A. S., Balboula, A. Z., Shrivastava, V., Ohring, J., and Schindler, K. (2014). Phosphorylation of threonine 3 on histone H3 by haspin kinase is required for meiosis I in mouse oocytes. J. Cell Sci. 127, 5066-5078. doi: $10.1242 /$ jcs. 158840

Niault, T., Hached, K., Sotillo, R., Sorger, P. K., Maro, B., Benezra, R., et al. (2007). Changing Mad2 levels affects chromosome segregation and spindle assembly checkpoint control in female mouse meiosis I. PLoS ONE 2:e1165. doi: 10.1371/journal.pone.0001165 
Ohkura, H. (2015). Meiosis: an overview of key differences from mitosis. Cold Spring Harb. Perspect. Biol. 7:a015859. doi: 10.1101/cshperspect.a015859

Ottolini, C. S., Newnham, L., Capalbo, A., Natesan, S. A., Joshi, H. A., Cimadomo, D., et al. (2015). Genome-wide maps of recombination and chromosome segregation in human oocytes and embryos show selection for maternal recombination rates. Nat. Genet. 47, 727-735. doi: 10.1038/ng.3306

Patel, J., Tan, S. L., Hartshorne, G. M., and McAinsh, A. D. (2015). Unique geometry of sister kinetochores in human oocytes during meiosis I may explain maternal age-associated increases in chromosomal abnormalities. Biol. Open 5, 178-184. doi: 10.1242/bio.016394

Perry, A. C., and Verlhac, M. H. (2008). Second meiotic arrest and exit in frogs and mice. EMBO Rep. 9, 246-251. doi: 10.1038/embor.2008.22

Rabitsch, K. P., Gregan, J., Schleiffer, A., Javerzat, J. P., Eisenhaber, F., and Nasmyth, K. (2004). Two fission yeast homologs of Drosophila Mei-S332 are required for chromosome segregation during meiosis I and II. Curr. Biol. 14, 287-301. doi: 10.1016/j.cub.2004.01.051

Revenkova, E., Herrmann, K., Adelfalk, C., and Jessberger, R. (2010). Oocyte cohesin expression restricted to predictyate stages provides full fertility and prevents aneuploidy. Curr. Biol. 20, 1529-1533. doi: 10.1016/j.cub.2010. 08.024

Ricke, R. M., Jeganathan, K. B., Malureanu, L., Harrison, A. M., and van Deursen, J. M. (2012). Bub1 kinase activity drives error correction and mitotic checkpoint control but not tumor suppression. J. Cell Biol. 199, 931-949. doi: $10.1083 /$ jcb.201205115

Riedel, C. G., Katis, V. L., Katou, Y., Mori, S., Itoh, T., Helmhart, W., et al. (2006). Protein phosphatase $2 \mathrm{~A}$ protects centromeric sister chromatid cohesion during meiosis I. Nature 441, 53-61. doi: 10.1038/nature04664

Sacristan, C., and Kops, G. J. (2015). Joined at the hip: kinetochores, microtubules, and spindle assembly checkpoint signaling. Trends Cell Biol. 25, 21-28. doi: 10.1016/j.tcb.2014.08.006

Sakuno, T., Tanaka, K., Hauf, S., and Watanabe, Y. (2011). Repositioning of aurora B promoted by chiasmata ensures sister chromatid monoorientation in meiosis I. Dev Cell. 21, 534-545. doi: 10.1016/j.devcel.2011. 08.012

Santaguida, S., Vernieri, C., Villa, F., Ciliberto, A., and Musacchio, A. (2011). Evidence that Aurora B is implicated in spindle checkpoint signalling independently of error correction. EMBO J. 30, 1508-1519. doi: 10.1038/emboj.2011.70

Sarangapani, K. K., Duro, E., Deng, Y., Alves Fde, L., Ye, Q., Opoku, K. N., et al. (2014). Sister kinetochores are mechanically fused during meiosis I in yeast. Science 346, 248-251. doi: 10.1126/science.1256729

Sasai, K., Katayama, H., Stenoien, D. L., Fujii, S., Honda, R., Kimura, M., et al. (2004). Aurora-C kinase is a novel chromosomal passenger protein that can complement Aurora-B kinase function in mitotic cells. Cell Motil Cytoskeleton 59, 249-263. doi: $10.1002 / \mathrm{cm} .20039$

Saurin, A. T., van der Waal, M. S., Medema, R. H., Lens, S. M., and Kops, G. J. (2011). Aurora B potentiates Mps1 activation to ensure rapid checkpoint establishment at the onset of mitosis. Nat. Commun. 2:316. doi: $10.1038 /$ ncomms 1319

Schindler, K., Davydenko, O., Fram, B., Lampson, M. A., and Schultz, R. M. (2012). Maternally recruited Aurora $\mathrm{C}$ kinase is more stable than Aurora B to support mouse oocyte maturation and early development. Proc. Natl. Acad. Sci. U.S.A. 109, E2215-E2222. doi: 10.1073/pnas.1120517109

Sebestova, J., Danylevska, A., Novakova, L., Kubelka, M., and Anger, M. (2012). Lack of response to unaligned chromosomes in mammalian female gametes. Cell Cycle 11, 3011-3018. doi: 10.4161/cc.21398

Shao, H., Li, R., Ma, C., Chen, E., and Liu, X. J. (2013). Xenopus oocyte meiosis lacks spindle assembly checkpoint control. J. Cell Biol. 201, 191-200. doi: $10.1083 /$ jcb. 201211041

Shepperd, L. A., Meadows, J. C., Sochaj, A. M., Lancaster, T. C., Zou, J., Buttrick, G. J., et al. (2012). Phosphodependent recruitment of Bub1 and Bub3 to Spc7/KNL1 by Mph1 kinase maintains the spindle checkpoint. Curr. Biol. 22, 891-899. doi: 10.1016/j.cub.2012.03.051

Shintomi, K., and Hirano, T. (2009). Releasing cohesin from chromosome arms in early mitosis: opposing actions of Wapl-Pds5 and Sgol. Genes Dev. 23, 2224-2236. doi: 10.1101/gad.1844309

Shirk, K., Jin, H., Giddings, T. H. Jr., Winey, M., and Yu, H. G. (2011). The Aurora kinase Ipll is necessary for spindle pole body cohesion during budding yeast meiosis. J. Cell Sci. 124(Pt 17), 2891-2896. doi: 10.1242/jcs. 086652

Shonn, M. A., McCarroll, R., and Murray, A. W. (2000). Requirement of the spindle checkpoint for proper chromosome segregation in budding yeast meiosis. Science 289, 300-303. doi: 10.1126/science.289.5477.300

Shonn, M. A., Murray, A. L., and Murray, A. W. (2003). Spindle checkpoint component Mad2 contributes to biorientation of homologous chromosomes. Curr. Biol. 13, 1979-1984. doi: 10.1016/j.cub.2003.10.057

Shrestha, R. L., Conti, D., Tamura, N., Braun, D., Ramalingam, R. A., Cieslinski, K., et al. (2017). Aurora-B kinase pathway controls the lateral to end-on conversion of kinetochore-microtubule attachments in human cells. Nat. Commun. 8:150. doi: 10.1038/s41467-017-00209-Z

Sliedrecht, T., Zhang, C., Shokat, K. M., and Kops, G. J. (2010). Chemical genetic inhibition of Mps1 in stable human cell lines reveals novel aspects of Mps1 function in mitosis. PLoS ONE 5:e10251. doi: 10.1371/journal.pone.0010251

Straight, A. F., Belmont, A. S., Robinett, C. C., and Murray, A. W. (1996). GFP tagging of budding yeast chromosomes reveals that protein-protein interactions can mediate sister chromatid cohesion. Curr. Biol. 6, 1599-1608. doi: 10.1016/S0960-9822(02)70783-5

Straight, P. D., Giddings, T. H. Jr., and Winey, M. (2000). Mps1p regulates meiotic spindle pole body duplication in addition to having novel roles during sporulation. Mol. Biol. Cell 11, 3525-3537. doi: 10.1091/mbc.11.10.3525

Sudakin, V., Chan, G. K., and Yen, T. J. (2001). Checkpoint inhibition of the APC/C in HeLa cells is mediated by a complex of BUBR1, BUB3, CDC20, and MAD2. J. Cell Biol. 154, 925-936. doi: 10.1083/jcb.200102093

Suijkerbuijk, S. J., Vleugel, M., Teixeira, A., and Kops, G. J. (2012). Integration of kinase and phosphatase activities by BUBR1 ensures formation of stable kinetochore-microtubule attachments. Dev. Cell 23, 745-755. doi: 10.1016/j.devcel.2012.09.005

Tachibana-Konwalski, K., Godwin, J., van der Weyden, L., Champion, L., Kudo, N. R., Adams, D. J., et al. (2010). Rec8-containing cohesin maintains bivalents without turnover during the growing phase of mouse oocytes. Genes Dev. 24, 2505-2516. doi: 10.1101/gad.605910

Tang, Z., Bharadwaj, R., Li, B., and Yu, H. (2001). Mad2-independent inhibition of APCCdc20 by the mitotic checkpoint protein BubR1. Dev. Cell 1, 227-237. doi: 10.1016/S1534-5807(01)00019-3

Tang, Z., Sun, Y., Harley, S. E., Zou, H., and Yu, H. (2004). Human Bub1 protects centromeric sister-chromatid cohesion through Shugoshin during mitosis Proc. Natl. Acad. Sci. U.S.A. 101, 18012-18017. doi: 10.1073/pnas.0408600102

Taylor, S. S., and McKeon, F. (1997). Kinetochore localization of murine Bub1 is required for normal mitotic timing and checkpoint response to spindle damage. Cell 89, 727-735. doi: 10.1016/S0092-8674(00)80255-X

Terret, M. E., Wassmann, K., Waizenegger, I., Maro, B., Peters, J. M., and Verlhac, M. H. (2003). The Meiosis I-to-Meiosis II transition in mouse oocytes requires separase activity. Curr. Biol. 13, 1797-1802. doi: 10.1016/j.cub.2003.09.032

Tighe, A., Staples, O., and Taylor, S. (2008). Mps1 kinase activity restrains anaphase during an unperturbed mitosis and targets Mad2 to kinetochores. J. Cell Biol. 181, 893-901. doi: $10.1083 /$ jcb.200712028

Touati, S. A., Buffin, E., Cladiere, D., Hached, K., Rachez, C., van Deursen, J. M., et al. (2015). Mouse oocytes depend on BubR1 for proper chromosome segregation but not for prophase I arrest. Nat. Commun. 6:6946. doi: $10.1038 /$ ncomms7946

Touati, S. A., and Wassmann, K. (2016). How oocytes try to get it right: spindle checkpoint control in meiosis. Chromosoma 125, 321-335. doi: 10.1007/s00412-015-0536-7

Tsuchiya, D., Gonzalez, C., and Lacefield, S. (2011). The spindle checkpoint protein Mad2 regulates APC/C activity during prometaphase and metaphase of meiosis I in Saccharomyces cerevisiae. Mol. Biol. Cell 22, 2848-2861. doi: 10.1091/mbc.E11-04-0378

Tsurumi, C., Hoffmann, S., Geley, S., Graeser, R., and Polanski, Z. (2004). The spindle assembly checkpoint is not essential for CSF arrest of mouse oocytes. J. Cell Biol. 167, 1037-1050. doi: 10.1083/jcb.200405165

Tunquist, B. J., Eyers, P. A., Chen, L. G., Lewellyn, A. L., and Maller, J. L. (2003). Spindle checkpoint proteins Mad1 and Mad2 are required for cytostatic factor-mediated metaphase arrest. J. Cell Biol. 163, 1231-1242. doi: $10.1083 /$ jcb. 200306153

Tunquist, B. J., Schwab, M. S., Chen, L. G., and Maller, J. L. (2002). The spindle checkpoint kinase bub1 and cyclin e/cdk2 both contribute to the establishment 
of meiotic metaphase arrest by cytostatic factor. Curr. Biol. 12, 1027-1033. doi: 10.1016/S0960-9822(02)00894-1

van der Horst, A., and Lens, S. M. (2014). Cell division: control of the chromosomal passenger complex in time and space. Chromosoma 123, 25-42. doi: 10.1007/s00412-013-0437-6

van der Waal, M. S., Saurin, A. T., Vromans, M. J., Vleugel, M., Wurzenberger, C., Gerlich, D. W., et al. (2012). Mps1 promotes rapid centromere accumulation of Aurora B. EMBO Rep. 13, 847-854. doi: 10.1038/embor.2012.93

Vaur, S., Cubizolles, F., Plane, G., Genier, S., Rabitsch, P. K., Gregan, J., et al. (2005). Control of Shugoshin function during fission-yeast meiosis. Curr. Biol. 15, 2263-2270. doi: 10.1016/j.cub.2005.11.034

Vázquez-Novelle, M. D., Sansregret, L., Dick, A. E., Smith, C. A., McAinsh, A. D., Gerlich, D. W., et al. (2014). Cdk1 inactivation terminates mitotic checkpoint surveillance and stabilizes kinetochore attachments in anaphase. Curr. Biol. 24, 638-645. doi: 10.1016/j.cub.2014.01.034

Vleugel, M., Hoek, T. A., Tromer, E., Sliedrecht, T., Groenewold, V., Omerzu, M., et al. (2015). Dissecting the roles of human BUB1 in the spindle assembly checkpoint. J. Cell Sci. 128, 2975-2982. doi: 10.1242/jcs. 169821

Wassmann, K., Niault, T., and Maro, B. (2003). Metaphase I arrest upon activation of the Mad2-dependent spindle checkpoint in mouse oocytes. Curr. Biol. 13, 1596-1608. doi: 10.1016/j.cub.2003.08.052

Weiss, E., and Winey, M. (1996). The Saccharomyces cerevisiae spindle pole body duplication gene MPS1 is part of a mitotic checkpoint. J. Cell Biol. 132, 111-123.

Williams, S. J., Abrieu, A., and Losada, A. (2016). Bub1 targeting to centromeres is sufficient for Sgol recruitment in the absence of kinetochores. Chromosoma 26, 279-286. doi: 10.1007/s00412-016-0592-

Winey, M., Goetsch, L., Baum, P., and Byers, B. (1991). MPS1 and MPS2: novel yeast genes defining distinct steps of spindle pole body duplication. J. Cell Biol. $114,745-754$.

Winey, M., Mamay, C. L., O’Toole, E. T., Mastronarde, D. N., Giddings, T. H. Jr., McDonald, K. L., et al. (1995). Three-dimensional ultrastructural analysis of the Saccharomyces cerevisiae mitotic spindle. J. Cell Biol. 129, 1601-1615. doi: $10.1083 /$ jcb.129.6.1601
Xu, P., Raetz, E. A., Kitagawa, M., Virshup, D. M., and Lee, S. H. (2013). BUBR1 recruits PP2A via the B56 family of targeting subunits to promote chromosome congression. Biol. Open 2, 479-486. doi: 10.1242/bio.20134051

Yamagishi, Y., Yang, C. H., Tanno, Y., and Watanabe, Y. (2012). MPS1/Mph1 phosphorylates the kinetochore protein KNL1/Spc7 to recruit SAC components. Nat. Cell Biol. 14, 746-752. doi: 10.1038/nc b2515

Yoshida, S., Kaido, M., and Kitajima, T. S. (2015). Inherent instability of correct kinetochore-microtubule attachments during meiosis I in oocytes. Dev. Cell 33, 589-602. doi: 10.1016/j.devcel.2015.04.020

Yu, H. G., and Koshland, D. (2007). The aurora kinase Ipl1 maintains the centromeric localization of PP2A to protect cohesin during meiosis. J. Cell Biol. 176, 911-918. doi: 10.1083/jcb.200609153

Zhang, X., Ma, C., Miller, A. L., Katbi, H. A., Bement, W. M., and Liu, X. J. (2008). Polar body emission requires a RhoA contractile ring and $\mathrm{Cdc42}$-mediated membrane protrusion. Dev. Cell 15, 386-400. doi: 10.1016/j.devcel.2008.07.005

Zielinska, A. P., Holubcova, Z., Blayney, M., Elder, K., and Schuh, M. (2015). Sister kinetochore splitting and precocious disintegration of bivalents could explain the maternal age effect. Elife 4:e11389. doi: 10.7554/eLife. 11389

Conflict of Interest Statement: The authors declare that the research was conducted in the absence of any commercial or financial relationships that could be construed as a potential conflict of interest.

The reviewer DF declared a shared affiliation, with no collaboration, with one of the authors, $\mathrm{KW}$, to the handling Editor.

Copyright $\odot 2017$ Marston and Wassmann. This is an open-access article distributed under the terms of the Creative Commons Attribution License (CC BY). The use, distribution or reproduction in other forums is permitted, provided the original author(s) or licensor are credited and that the original publication in this journal is cited, in accordance with accepted academic practice. No use, distribution or reproduction is permitted which does not comply with these terms. 